\title{
Euripides in der kaiserzeitlichen Stoa, Skepsis und im Neuplatonismus
}

\begin{abstract}
This paper examines the reception of Euripides in some philosophical schools of the Imperial Era and Late Antiquity on the basis of some of their most important representatives. It is shown that Epictetus mainly cites Euripidean figures as an example of negative behaviour in order to "correct" it in the light of Stoic philosophy, while Marcus Aurelius primarily uses Euripidean gnomai to confirm his own statements. It is further explained that Sextus Empiricus follows an Epicurean critique of the argumentative use of quotations from the poets which is directed against the Stoa and contemporary grammarians, while he himself uses some quotations from Euripides affirmatively for his own ethics, again following an Epicurean source. Finally, it is shown that since Proclus, who systematized Plato's partly ambivalent statements on poetry and integrated poetry into a Neoplatonic system of knowledge, the Neoplatonists used Euripides for the pedagogical transfer of knowledge in philosophical beginners' instruction.
\end{abstract}

In der Kaiserzeit wurde Euripides gerne als „Weiser“ (бoழóৎ) oder „Bühnenphilosoph“

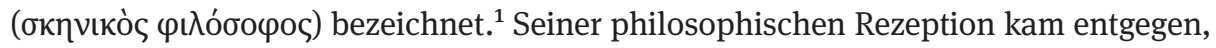
dass er, gerade in den Sprechpartien, häufig Gnomen verwendete und intensiv Themen und Thesen von Sophisten und zeitgenössischen Philosophen zu Wort kommen ließ. ${ }^{2}$ Bereits Aristophanes sagte Euripides eine starke Affinität zur Sophistik und besonders zu Sokrates nach. ${ }^{3}$ Nach Diogenes Laertios hielt Protagoras seine Vorlesungen in Athen im Hause des Euripides oder in dem des Megakleides (Diog. Laert. 9,54), und Euripides und Sokrates seien beide Schüler des Anaxagoras gewesen $(2,45) .{ }^{4}$ Diogenes Laertios beginnt sein Kapitel über Sokrates damit, dass es die Ansicht gebe, dieser habe an den Dichtungen des Euripides „mitgedichtet“ (2,18). Er zitiert hierzu, allerdings korrupt überliefert und mit falscher Autorenzuordnung, den Komödiendichter Telekleides, wonach Sokrates für ein neues Stück des Euripides das

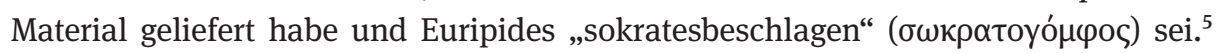

1 Vgl. Kannicht 2004, 120-123 (T 158-169).

2 Vgl. Egli 2003; Most 2003.

3 Vgl. Ar. Ra. 954-958. 1491-1495.

4 Vgl. Marm. Par. 60 = FGrH 239 A 60 = DK 59 A 4a; Schol. Eur. I,1,10 Schwartz.

5 Diogenes Laertios überliefert das Wortspiel, das neue Stück des Euripides seien die Phryger und Sokrates habe dazu das Reisig ( $\varphi$ vóyava) geliefert; dieses Wort schreibt er einem Mnesilochos zu, den er vermutlich mit dem Komödiendichter Mnesimachos verwechselt. Der Vers des Telekleides besagte hingegen, dass Mnesilochos der sei, der dem Euripides ein neues Stück geröstet habe ( $\varphi \rho u ́ y \varepsilon ı)$, und 
Schließlich zitiert Diogenes ein Distichon des Aristophanes aus der ersten Fassung seiner Wolken, Euripides habe geschwätzige und sophistische Tragödien geschrieben (Ar. fr. 392 Kassel-Austin). Diogenes sammelt hier allerlei biographisches Material, das seit der attischen Komödie auf anekdotische Weise über die Beziehung des Euripides zur Sokratik entstanden war - eine Tradition, die weiterreichte bis zu Nietzsche, der noch als Student Studien zu den Quellen des Diogenes Laertios anstellte und in seiner 1872 erschienenen „Geburt der Tragödie“ Euripides als von Sokrates beeinflusste Verfallsform der Tragödie ansah. ${ }^{6}$

Der folgende Beitrag möchte sich nicht auf diese biographischen Zurechnungen des Euripides zur Philosophie konzentrieren, sondern darauf, wie verschiedene Philosophenschulen der Kaiserzeit Sentenzen oder philosophische Ansichten, die Figuren euripideischer Dramen äußerten, als autoritative Meinung des Euripides zur Bestätigung eigener philosophischer Ansichten benutzten und in das eigene philosophische Denken integrierten. Im Fokus sollen mit der Stoa, der Skepsis und dem Neuplatonismus die kaiserzeitlichen Schulen stehen, die am häufigsten und weitreichendsten Euripides als philosophische Autorität benutzten. Im kaiserzeitlichen Aristotelismus und Kynismus lassen sich keine Euripides-Zitate finden. ${ }^{7}$

\section{Stoa}

\subsection{Epiktet}

Im Zentrum von Chrysipps Euripides-Rezeption steht dessen Medea. So heißt es über eine seiner Schriften, dass er darin beinahe die gesamte Medea des Euripides zitiert habe, so dass diese Schrift ironisch von einem Leser als „Medea des Chrysipp“ bezeichnet worden sei (Diog. Laert. 7,180). ${ }^{8}$ Besonders der Ausspruch der Medea „Ich weiß, welche Übel ich zu tun im Begriff bin, aber der Zorn ist stärker als meine Vernunft“ (Eur. Med. 1078f.) forderte die Philosophen heraus und ließ Medea zum Paradebeispiel in der Erörterung des Problems der Willensschwäche ( $\alpha$ k $\left.\rho \alpha \sigma^{\prime} \alpha\right)$ werden. Chrysipp und andere Stoiker scheinen diese Stelle als Beweis dafür angesehen zu

dass ihm Sokrates dazu das Reisig geliefert habe (vgl. Schol. Eur. I,1,12-2,2 Schwartz; Telecl. fr. 41 Kassel-Austin; das nächste Zitat, Euripides sei „sokratesbeschlagen“, ist hier fr. 42).

6 Die drei Studien zu Diogenes Laertios sind zwischen 1868 und 1870 erschienen (,De Laertii Diogenis fontibus“, „Analecta Laertiana“ und „Beiträge zur Quellenkunde und Kritik des Laertius Diogenes“, (vgl. Nietzsche 1982, 77-245). Zur Euripides-Kritik in der „Geburt der Tragödie“ vgl. Nietzsche 1972, 71-84 (cp. 11f.). Prägnant bezeichnete Nietzsche Euripides als „Dichter des aesthetischen Sokratismus“ (Nietzsche 1972, 83): „dessen oberstes Gesetz ungefähr so lautet: ,alles muss verständig sein, um schön zu sein'; als Parallele zu dem sokratischen ,nur der Wissende ist tugendhaft"“ (Nietzsche 1972, 81).

$7 \mathrm{Zu}$ einem Vertreter des Mittelplatonismus, nämlich Plutarch, siehe oben den Beitrag von Jan Opsomer in diesem Band.

8 Zur Praxis des Chrysipp, ausgiebig Dichterzitate zu verwenden, vgl. SVF 2,883f.; 906 f. 
haben, dass alle willentlichen Handlungen (das schließt auch schlechte Handlungen ein) von der ganzen Seele ausgeführt werden, während die Platoniker sie als Beweis für die Existenz eines nicht-rationalen Seelenteils ansahen, der in Widerspruch zum rationalen Seelenteil geraten kann. ${ }^{9}$

Epiktet dürfte seine Kenntnis der euripideischen Medea ganz aus Chrysipps $\mathrm{Me}$ dea und eine gewisse Orientierung über ihre Handlung aus Dramenhypothesen bezogen haben. ${ }^{10}$ Allgemein führt er Medea wie auch andere Figuren der Tragödien ausschließlich als Beispiele negativen Verhaltens an, und zwar weit seltener als etwa Sokrates, Diogenes, Odysseus und Herakles, die er als positive Beispiele richtigen Verhaltens verwendet. ${ }^{11}$ Das oben genannte Medea-Zitat ist eingebettet in einen Kontext, in dem behauptet wird, dass es unmöglich sei, einer falschen Vorstellung zuzustimmen, und es im selben Maße auch unmöglich sei zu handeln, wenn man die Handlung nicht für geboten oder nützlich hält (Epict. Diss. 1,28,1-6). Nach stoischer

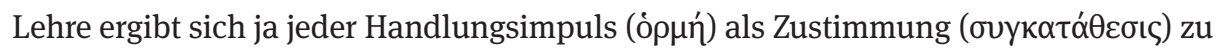
einer bestimmten Vorstellung $(\varphi \alpha v \tau \alpha \sigma i \alpha){ }^{12}$ Medea erschien es, so Epiktet, also nützlicher, ihrem Zorn zu folgen und sich an ihrem Mann zu rächen, als ihre gemeinsamen Kinder zu retten (1,28,7). Zwar habe sie sich hierin getäuscht, aber solange ihr niemand gezeigt habe, dass sie sich täusche, und sie nur ihrer Vorstellung gefolgt sei, dürfe man ihr nicht übelnehmen, dass sie sich in schwerem Irrtum befunden habe, sondern müsse sie bemitleiden wie einen Blinden oder Lahmen $(1,28,8 \mathrm{f}.){ }^{13}$ Wenn Euripides die Medea als dramatischen Einwand gegen den sokratischen Intellektualismus konzipiert hat, also vor allem gegen die These, dass niemand freiwillig Unrecht tue, ${ }^{14}$ dann korrigiert Epiktet diesen Einwand, indem er an der sokratischen These, gestützt auf die stoische Handlungstheorie, festhält.

Außerdem unterwirft er die Tragödie einem heteronomen, außer-ästhetischem Blickwinkel und versteht sie als dramatischen Ausdruck eines bestimmten Vorstellungsgebrauchs. So definiert er die Tragödie allgemein als „in bestimmtem Versmaß abgefasste Vorführung der Leiden von Menschen, die äußere Dinge bewundern“ $(1,4,26) .{ }^{15}$ Der Phoinix und der Hippolytos des Euripides oder der Ödipus des Sophokles

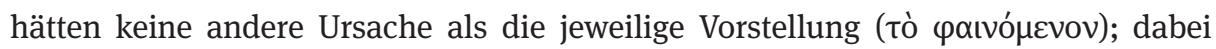
würden die Dramenprotagonisten hierauf keine Sorge verwenden und wie Verrückte jeder beliebigen Vorstellung folgen, wovon unser gewöhnliches Verhalten aber gar nicht sehr abweichen würde (1,28,32f.). Epiktet gesteht dem Rezipienten von Tragödien also als erste unmittelbare Reaktion Verständnis für die Situation der Protago-

9 Das hat Dillon 1997 gezeigt.

10 Vgl. Bonhöffer 1898, 4.

11 Vgl. Wehner 2000, 222-232.

12 Vgl. SVF 2,52; 3,169, 40,17-20; 3,177, 42,35f.

$13 \mathrm{Zu}$ dieser Stelle vgl. Dobbin 1998, 220 -223; Wehner 2000, $232 \mathrm{f}$.

14 Vgl. Dodds 1951, 186f.; auch Snell 1975, 119-121.

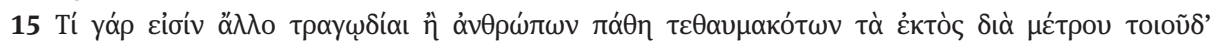
$\varepsilon \dot{\pi} \delta \varepsilon เ \kappa v u ́ \mu \varepsilon v \alpha$; 
nisten bis hin sogar zu Mitleid zu, weil sich im Prinzip alle Menschen so verhalten wie die Tragödienhelden. Doch solle er nicht dabei stehenbleiben, sondern in kritischer Distanzierung zur Erkenntnis gelangen, dass sich die Protagonisten wie Verrückte, Blinde oder Lahme verhalten und ihnen der richtige Gebrauch der Vorstellungen

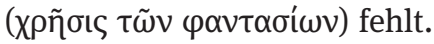

Wieder anhand der Medea zeigt Epiktet, wie er sich die richtige distanznehmende Beurteilung der Tragödie denkt. In einem Kontext, in dem es um die Frage geht, wie man seine Vorerwartungen an die gegebene Situation anpassen kann, kritisiert er Medea dafür, dass sie aus der richtigen Erkenntnis, dass ihre Jason betreffenden Wünsche nicht wahr geworden sind, den Entschluss abgeleitet habe, den Mann zu bestrafen, die Kinder zu töten und damit auch sich selbst zu bestrafen (2,17,19f.). ${ }^{16}$ Er beschreibt sie hin-

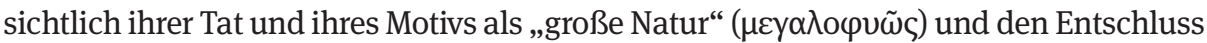

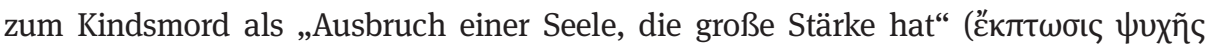

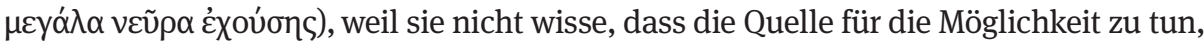
was man wünsche, nicht in prinzipiell veränderlichen äußeren Faktoren liege, sondern in ihr selbst $(2,17,19$. 21). So hatte er ja auch nach der Definition der Tragödie ihre ethische Funktion bestimmt: Durch ihre Vorführung der Leiden von Menschen aufgrund ihrer Bewunderung äußerer Dinge solle beim Rezipienten die Erkenntnis erwachsen,

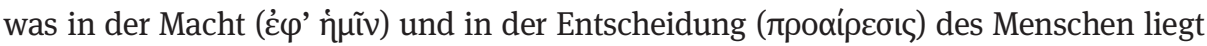
und was nicht, eine Erkenntnis, aus der wiederum Glück (عű $\rho \circ \alpha)$ und Seelenruhe $\left(\dot{\alpha} \tau \alpha \rho \alpha \xi^{\prime} \alpha\right)$ folgen $(1,4,27) .{ }^{17}$ Er spricht Medea direkt mit folgendem Rat an:

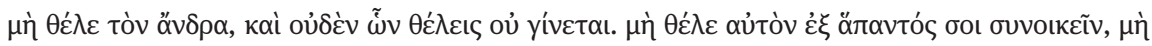

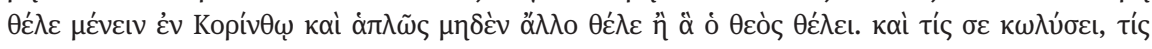

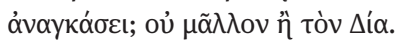

Wolle nicht den Mann, und nichts von dem, was du willst, geschieht nicht! Wolle nicht, dass er um jeden Preis mit dir zusammenlebt, wolle nicht in Korinth bleiben und wolle schlechthin nichts anderes, als was Gott will. Und wer wird dich hindern, wer zwingen? Keiner, außer Zeus. $(2,17,22)^{18}$

Epiktet tritt hier in einen inneren Dialog mit Medea, in den auch der Rezipient der Tragödie - gemeint ist insbesondere der Schüler Epiktets - treten soll: ${ }^{19}$ Er soll Medea zurechtweisen wie ein Arzt, der einen Verrückten heilen soll. ${ }^{20}$ Epiktet rekurriert

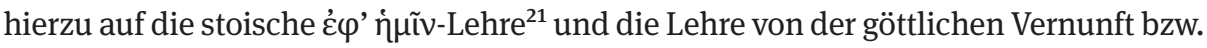

16 Vgl. Eur. Med. 772-810; 1021-1080.

17 Eỉ yà

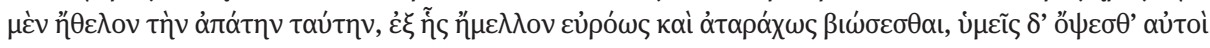
Tí $\theta \varepsilon ́ \lambda \varepsilon \tau \varepsilon$.

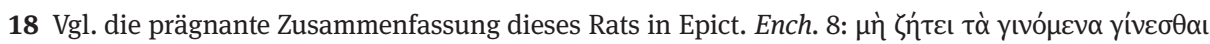

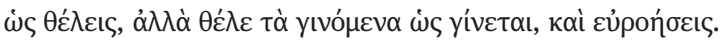

19 Die Dialogpartien stellt Wehner 2000 als charakteristisches Merkmal der Diatriben heraus.

20 Vgl. Nussbaum 1993, $142 \mathrm{f}$.

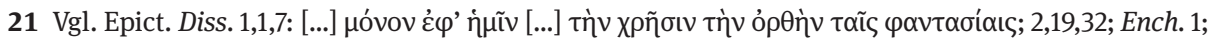

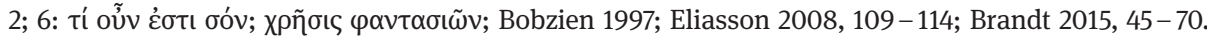


dem Naturgesetz als leitendem Handlungsprinzip ${ }^{22}$ und empfiehlt, der falschen Vorstellung nicht zuzustimmen und diese durch eine richtige zu ersetzen. Das setzt allerdings schon eine entsprechende Übung voraus; ${ }^{23}$ Medea hätte also eine stoische Weise sein oder zumindest auf dem Weg dorthin sein müssen, um zu lernen, sich in ihr Schicksal zu fügen. ${ }^{24}$

In seinem Encheiridion formuliert Epiktet genau dies - sich freiwillig in sein Schicksal zu fügen - als Ziel seines Übungsbuchs. Bemerkenswert ist, dass er dieses Ziel nicht in eigenen Worten, sondern durch eine nicht durch Zwischentexte oder Benennung der Quelle unterbrochene Reihe von Zitaten ausdrückt, die aus Kleanthes' Zeushymnos, aus einem nicht überlieferten Stück des Euripides und aus Platons Apologie und Kriton stammen, also zwei Zitaten im iambischen Versmaß und zwei Prosazitaten; sie sind besonders auffällig, da es im gesamten vorangegangenen Text keine Zitate gibt. Das zeigt, dass mit den Zitaten, insbesondere mit dem des KleanthesHymnos, eine deutliche Schlusswirkung und in der Art einer Sphragis eine autoritative Beglaubigung der zuvor dargelegten Meditationen und Übungsanleitungen erzielt werden soll.

Erstaunlich ist hier auch die Reihe der Zitate und der Zitatautoritäten: Der Zeushymnos des Kleanthes wird von Epiktet häufig auch in den Diatriben zitiert. ${ }^{25}$ Ebenso häufig benutzt er auch das Zitat aus Platons Kriton (43d7f.) „wenn es den Göttern gefällt, so sei es“, mit dem Sokrates sein Todesurteil akzeptiert, und das Zitat aus der Apologie (30c6f.), dass Anytos und Meletos ihn zwar töten, ihm aber nicht schaden könnten. ${ }^{26}$ Sokrates hat für Epiktet die höchste Bedeutung als Leitbild ethisch richtigen Verhaltens, mit beiden Zitaten soll Sokrates zum Ideal des stoischen Weisen

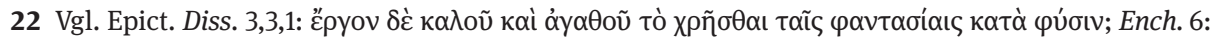

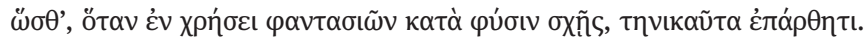

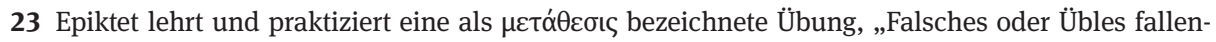
zulassen und durch Richtiges oder Gutes zu ersetzen“ (Brandt 2015, 25f.). Sein Rat an Medea, dem

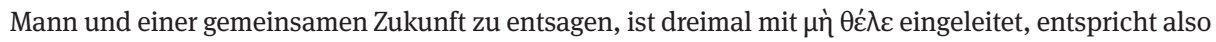

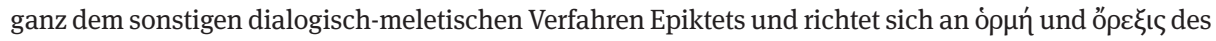
fiktiven Gegenübers Medea.

24 Weitere Euripides-Figuren, allerdings weniger prominent als Medea, die Epiktet als Beispiel negativen Verhaltens mit entsprechenden wörtlichen Zitaten anführt, sind Admets Vater Pheres aus der Alkestis, der nicht bereit ist, den todgeweihten Sohn durch den eigenen Tod zu retten (mit Eur. Alc. 691, zitiert Epict. Diss. 2,22,11) und das Brüderpaar Eteokles und Polyneikes, die sich nach dem Leben trachten (Eur. Phoen. 621 f., zitiert Epict. Diss. 2,22,14); beide Zitate stehen in der Behandlung der Frage, was Freundschaft $(\varphi \iota \lambda i \alpha)$ ist.

25 Zur Häufigkeit und den Stellen bei Epiktet vgl. Brandt 2015, 319. Zur griechischen und lateinischen Rezeption dieser hier zitierten vier Verse bis in die byzantinische Zeit (u. a. bei Sen. Ep. 107,11 in lateinischer Übersetzung) vgl. Perkams 2005.

26 In den Diatriben kommen einmal $(3,22,95)$ der erste Kleanthes-Vers und das Kriton-Zitat hintereinander. Im Encheiridion hat dessen Herausgeber diese wohl um das Euripides- und das Apologie-Zitat ergänzt (vgl. Wehner 2000, 121). 
stilisiert werden. ${ }^{27}$ Singulär stehen bei Epiktet hingegen die beiden Euripides-Verse „Wer sich der Notwendigkeit auf rechte Weise fügt, ist bei uns weise und kennt das Göttliche“ (fr. 965,5f. Kannicht). Epiktet war sich gewiss nicht bewusst, dass Kleanthes mit seinen Versen einige Verse aus Euripides' Hekuba adaptierte und es daher kein Zufall sein kann, dass im Anschluss an das Zitat des Zeushymnos Euripides zitiert wird. ${ }^{28}$ Das Verbindende aller drei Zitate ist die Fügsamkeit des Weisen gegenüber dem Göttlichen und dem Schicksal. Das Euripides-Zitat stellt als einziges die Notwendig-

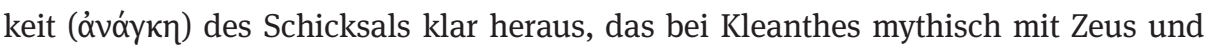
Pepromene und bei Platon allgemein mit dem Göttlichen umschrieben ist. Dabei ist die Notwendigkeit des Schicksals, das in der Tragödie zwar als unentrinnbar, aber nicht immer als vernünftig und gerecht erfahren wird, klar positiv im stoischen Sinne des den gesamten Kosmos vernünftig ordnenden Fatums umgedeutet. ${ }^{29}$ Es ist sogar möglich, dass Epiktet den Euripides-Text im Hinblick auf seine ethische Hauptaussage zugespitzt und textlich leicht umgeformt hat, denn bei Plutarch (Cons. ad Apoll.116F)

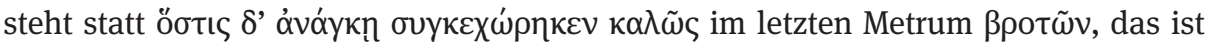
vermutlich der originale Text. ${ }^{30}$ Aber erst zusammen ergeben die vier Zitate, indem sie sich wechselseitig erläutern, die beabsichtigte Schlussbotschaft des Encheiridion.

Bemerkenswert ist auch Epiktets Umgang mit dem Zitat aus Euripides' Kresphontes, dass man die Neugeborenen beklagen soll angesichts der Übel, die ihnen in ihrem Leben noch bevorstehen, und sich mit den Toten freuen und sie preisen solle, weil für sie das Leiden beendet sei (fr. 449,3-6 Kannicht). Epiktet verwendet die Elemente dieses Zitats, das bei Sextus Empiricus zustimmend zitiert werden wird, ${ }^{31}$ und wendet sie gegen dessen ursprüngliche Intention: Man müsse angesichts bevorstehender Übel nicht den Lebenden oder den Toten beklagen, sondern den, der, noch am Leben, das ihm als Mensch Eigene verliere, nämlich die Anlagen des Verstandes in ihm (Diss. 4,5,15f.). Epiktet nimmt also der pessimistischen Klage über das Leben an sich die Spitze zugunsten der Kritik an einer bestimmten Lebensform, die das Wesen des Menschen und das summum bonum des menschlichen Lebens verfehlt,

27 Vgl. Döring 1974; Wehner 2000, 226-230. 238-240; Gourinat 2001, 137-165; Long 2002; Brandt 2015, 323f. (auch zu Häufigkeit und Stellen sowie weiterer Literatur Anm. 1488f.).

28 Vgl. Brandt 2015, 322f. Zuerst hat den Bezug des Zeushymnos zur Hekuba festgestellt Praechter 1899 (weitere Literatur bei Brandt 2015, 323 Anm. 1485, außerdem Dalfen 1971).

29 Vgl. Brandt 2015, 322.

30 Vgl. Nauck/Snell 1964, 672; Kannicht 2004, 964 f.; Perkams 2005, 61. Ein anderes, weit prägnanteres

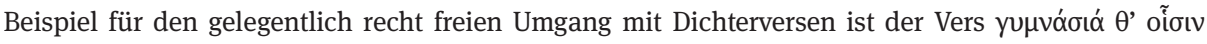

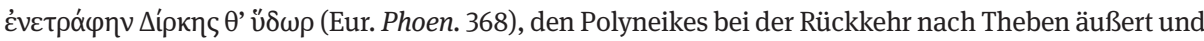
den Epiktet parodiert als Beispiel für jemanden, der sich über das Fehlen gewohnter Lebensumstände beklagt, wenn er sich etwa an das gute Wasser, das das Marcia-Aquädukt nach Rom bringt, gewöhnt hat und bei dessen Ausbleiben einen „Vers, ähnlich dem des Euripides“, vorbringt, nämlich „die

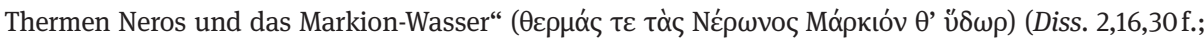
vgl. Wehner 2000, 52 mit Anm. 123).

31 Vgl. unten S. 314. 
wie es die Stoiker definieren. Das Zitat ist kein rhetorischer Schmuck, ${ }^{32}$ sondern fungiert innerhalb der philosophischen Argumentation als Antithese zur eigentlich intendierten Aussage. Die dadurch ausgedrückte Kritik an dem Zitatgehalt wirkt zurück auf diesen und korrigiert ihn zugunsten der stoischen Anthropologie und Ethik.

\subsection{Mark Aurel}

Ähnlich wie Epiktet betont auch Mark Aurel in seinen Selbstbetrachtungen, die von Epiktet stark beeinflusst sind, ${ }^{33}$ den moralischen Nutzen vor dem ästhetischen Vergnügen als Zweck der Tragödie:

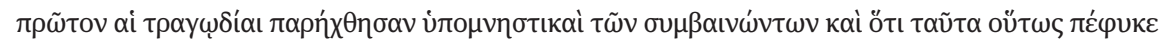

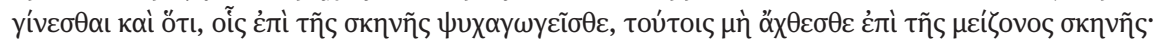
ó $\alpha \tilde{\tau} \tau \varepsilon$ yà KıӨ

Zuerst wurden die Tragödien aufgeführt als Erinnerung daran, was geschieht und dass sich dies von Natur aus so ereignet und dass ihr euch nicht über das, wovon ihr auf der Bühne bewegt werdet, auf der größeren Bühne (sc. der Welt) ärgert. Denn ihr seht, dass diese (sc. Geschehnisse) so zu Ende kommen müssen und dass diese auch die, die „Oh Kithairon“ rufen, ertragen. (Marc. Aur. 11,6,1f.)

Zwar kennt auch Mark Aurel das ästhetische Vergnügen sowohl an der Schönheit von Begleiterscheinungen natürlicher Prozesse (z. B. das beim Backen an einigen Stellen geplatzte Brot oder die runzlige Stirn eines Löwen) als auch an der Schönheit von künstlerischen Nachahmungen natürlicher Prozesse. ${ }^{34}$ Doch vorrangig ist für ihn der moralisch-didaktische Zweck der Tragödie: Ausgehend von der auch bei Epiktet zu findenden Parallelisierung des menschlichen Lebens und der Geschichte mit dem Drama, ${ }^{35}$ wird die Tragödie primär verstanden als „Erinnerung an Geschehnisse“ (ن่Tó $\mu \nu \eta \mu \alpha \tau \tilde{\omega} v \sigma u \mu \beta \alpha \iota v o ́ v \tau \omega v)$.

Vergleicht man diese Definition etwa mit der aristotelischen Definition der Tra-

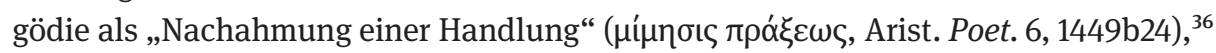

32 Dafür spricht sich Wehner 2000, 220 aus.

33 Zum Titel vgl. van Ackeren 2011, 49-51; zum Einfluss Epiktets vgl. Dalfen 1967, 82-160; van Ackeren 2011, 180 - 204.

34 Vgl. 3,2, bes. 3,2,4 (mit demselben Ausdruck für die innere Bewegung der Seele aufgrund einer

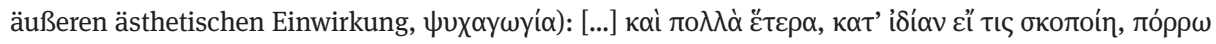

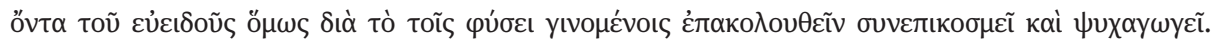

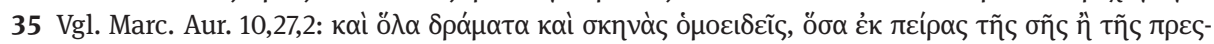

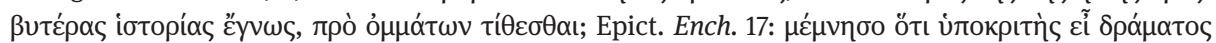

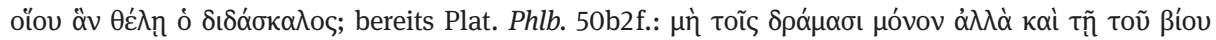

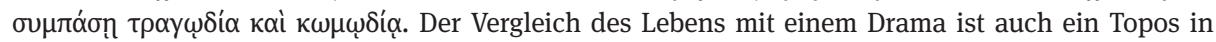
kynischen Diatriben (vgl. Brandt 2015, 139 mit Anm. 597).

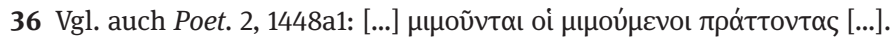


fällt auf, dass Mark Aurel nicht von Handlungen und entsprechend von freien nach eigenen Gründen und Motiven handelnden Akteuren von Handlungen spricht, sondern gleichsam entpersonalisiert von „Geschehnissen“ oder „Ereignissen“, gemäß seiner stoischen Überzeugung, dass es für alle Wesen notwendig ist, dem Weltlauf zu folgen, der Mensch als vernunftbegabtes Lebewesen dies allerdings freiwillig tun kann (Marc. Aur. 10,28,2). ${ }^{37}$ Auch im Vergleich zu Epiktet, der die Tragödie als „Vorführung der Leiden von Menschen, die äußere Dinge bewundern“ (Epict. Diss. 1,4,26) definiert, fällt auf, dass er nicht den leidenden Menschen als Handlungsakteur oder moralisches Fehlverhalten im Blick hat, das es zu didaktischen Übungszwecken gleichsam ex post zu korrigieren gilt, sondern vom Standpunkt des stoischen Weisen, der bereits die $\pi \alpha ́ \theta \eta$ - das Leiden und die Leidenschaften - zugunsten des stoischen Ideals der ,Leidenslosigkeit‘ ( $\alpha \dot{\alpha} \alpha \dot{\theta} \iota \alpha)$ abgelegt und sich freiwillig in das Schicksal gefügt hat.

Daher sind die Tragödien als Kunstwerke für ihn nicht primär wie für Aristoteles Nachahmungen $\left(\mu \iota \eta^{\prime} \mu \alpha \tau \alpha\right)$ natürlicher Prozesse, obwohl er auch diesen Ausdruck etwa für bildnerische Kunstwerke verwendet, ${ }^{38}$ oder wie für Epiktet dramatisch-rhe-

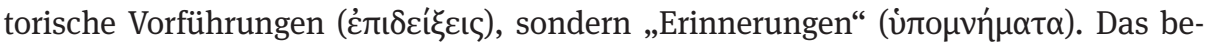
deutet einerseits eine zutiefst pessimistische Sicht auf das Leben, da die Zuschauer die in der Tragödie dargestellten Ereignisse (z. B. Inzest, Kindsmord, Ehebruch u. a.) anscheinend alle aus eigener Anschauung oder zumindest vom Hörensagen kennen und durch die Tragödie nur daran ,erinnert' werden. Andererseits zeigt dieser Ausdruck klar eine didaktische Zielsetzung an, wenn man etwa daran denkt, dass Polybios das Ziel seiner Geschichtsschreibung darin sah, den Leser durch die „Erinnerung fremder

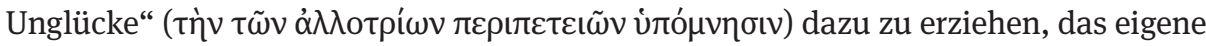
Schicksal edel zu ertragen (Plb. 1,1,2). ${ }^{39}$ Allerdings gilt diese Didaxe nicht einem realen oder imaginierten Schüler, sondern nur sich selbst. Und so bezeichnet Mark Aurel seine eigenen Aufzeichnungen als nichts anderes denn als „kleine Erinnerungsstücke“ oder „Kommentare“ (ن்

Das obige Zitat legt nahe, dass er die Tragödienprotagonisten als Exempla ansieht, anders als Epiktet aber nicht für negatives Verhalten, das durch philosophische Paränese korrigiert werden müsste, sondern als positives Rollenmodell dafür, das eigene, unabänderliche Schicksal edel und freiwillig zu ertragen. Er macht auch in seiner Schrift reichen Gebrauch von Exempla, etwa im ersten Buch von Verwandten

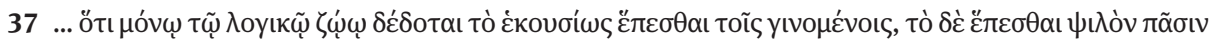

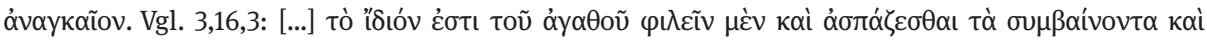

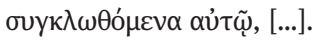

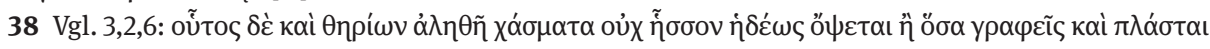

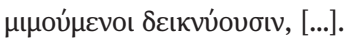

39 Darauf weist Farquharson 1944, 861 hin.

40 Zur Textsorte „Kommentar“ vgl. Dickey 2007, 11-14. Wenn die Tragödie, wie oben gesehen, als „ல்тó $\mu v \eta \mu \alpha$ der Geschehnisse“ bestimmt wird, kann man das auch im Sinne eines erinnernden und reflektierenden Kommentars zu den exemplarischen Geschehnissen des menschlichen Lebens verstehen. 
und Lehrern, die ihn bestimmte Tugenden gelehrt hätten, aber auch von historischen Persönlichkeiten oder Philosophen..$^{41}$ Figuren der Tragödie und somit der Bezug auf konkrete Tragödienhandlungen kommen aber bei ihm, anders als bei Epiktet, trotz der allgemeinen Wertschätzung der Tragödie als moralisches Vorbild für die Einübung des richtigen Verhaltens angesichts des Schicksals, nicht vor. Vielmehr arbeitet er mit bestimmten Gnomen und Chrien, ohne den Autor oder genauen Herkunftskontext anzugeben. Auch die Lese- oder Bildungsfrüchte fremder Autoren dienen wie die anderen Merksätze der Selbstbetrachtungen der „Erinnerung an die Freiheit von Schmerz und Furcht“ (10,34,1); sie rekapitulieren also nicht bloß Gelesenes und Bekanntes, sondern rufen den Schreiber paränetisch zur Verwirklichung des eigenen ethischen Ziels auf. Sie dienen - wie auch die anderen Merksätze - als Hilfe (ßoń $\eta \eta \mu \alpha$, 4,50), Leitfaden ( $\alpha \nu \omega \dot{\omega}, 5,22.10,2)$ oder lebendige Lehrsätze $(7,2) .{ }^{42}$ Vom übergroßen „Hunger nach Büchern“ möge man sich befreien (2,3). Doch bedeutet das nicht, dass der literarischen oder philosophischen Bildung, wie sie etwa in den Zitaten zum Ausdruck kommt, keine Bedeutung zukommt, wenn sie denn als Paränese zum ethisch richtigen Ziel eingesetzt wird.

Euripides ist nun der einzige von Mark Aurel zitierte Tragödienautor, ${ }^{43}$ an Quantität der Dichterzitate kommen nur Homer, Hesiod und Menander dem Euripides gleich. ${ }^{44}$ Im Anschluss an die eben zitierte Definition und Zielbestimmung der Tragödie führt er - ohne Nennung des Autors und des Quellorts - drei Sentenzen des Euripides als Beispiel dafür an, dass Sentenzen der Tragödiendichter „nützlich“ sind: „Wenn ich und mein Kind von den Göttern vernachlässigt wurden, dann hat auch dies einen Grund“ (Eur. Antiopa fr. 208,1f. Kannicht); „man darf den Dingen nicht zürnen“ (Eur. Bellerophontes fr. 287,1 Kannicht), ${ }^{45}$ und: ,,das Leben ernten wie eine reife Ähre“ (Eur. Hypsipyle fr. 757,925 Kannicht). Diese drei Zitate hat er bereits an einer früheren Stelle in enger Nachbarschaft zueinander benutzt, allerdings in leicht abgewandelter Reihenfolge (Marc. Aur. 7,41. 38. 40), bei den letzten beiden mit einer Fortsetzung des Zitats und gefolgt von einem weiteren, hier nicht genannten Euripides-Zitat (7,42 mit Eur. fr. 918 Kannicht). Das bedeutet, dass sich Mark Aurel vermutlich einer stoischen Sentenzensammlung bediente, in der die Zitate vermutlich als Zitatcluster überliefert waren, vielleicht unter einer Überschrift wie „Über den Gleichmut oder die Affektlo-

41 An historischen Persönlichkeiten z.B. Alexander, Pompeius, Caesar, an Philosophen Heraklit, Pythagoras, Sokrates (3,3; 6,47). Zum Exempla-Gebrauch bei Mark Aurel vgl. van Ackeren 2011, 53. 59-80. 300. 305-307.

42 Vgl. van Ackeren 2011, $292 \mathrm{f}$.

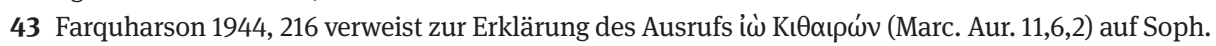
OT 1391. Das kann im Kontext eines Helden, der für das Ertragen von Leiden gelobt wird, durchaus Ödipus, der bekannteste Leidensmann der klassischen Tragödie, sein. Allerdings erscheint das Zitatsegment zu knapp und unspezifisch, um als eindeutiger Beweis für den Verweis auf Sophokles zu überzeugen; andere Tragödienheroen, die diesen Ausruf tätigen, sind denkbar.

44 Vgl. den Testimonien-Index bei Farquharson 1944.

45 Nach Stob. 4,108,39 dem Bellerophontes zugewiesen. 
sigkeit angesichts äußerer Ereignisse“. ${ }^{46}$ Weitere Euripides-Zitate belegen den Rat, den beständigen Wandel der Elemente zu bedenken (Marc. Aur. 7,47 mit 7,50 = Eur. Chrysippus fr. 839,9-11 Kannicht), sowie den Rat, nicht am Leben zu hängen und nicht durch Speisen, Getränke oder Zauberei den Tod abwenden zu wollen (Marc. Aur. 7,46 mit 7,51 = Eur. Suppl. 1110). Außerdem gibt es zweimal einen Verweis auf die Aussage, dass unser Geist der Gott in uns sei (Eur. fr. 1018 Kannicht; Marc. Aur. 5,27,2; 12,26,2), ein sehr beliebtes Zitat, das auch Menander zugeschrieben wurde. ${ }^{47}$

\section{Pyrrhonische Skepsis: Sextus Empiricus}

Euripides ist eine Hauptquelle auch für die pyrrhonische Skepsis. In einer Liste unbekannter Provenienz von Vorläufern der pyrrhonischen Skepsis werden bei Diogenes Laertios neben Homer, den Sieben Weisen, Xenophanes, Zenon von Elea und Demokrit auch Archilochos und Euripides mit Zitaten genannt, dann Xenophanes, Zenon von Elea, Demokrit, Platon, wieder Euripides, Empedokles, Heraklit und schließlich wieder Homer (Diog. Laert. 9,71-73). Hier ist grob eine Zweiteilung in Gruppen von Dichtern und Philosophen angedeutet. ${ }^{48}$ Klar ist durch diese Liste, dass für die Skepsis sowohl Dichtung als auch Philosophie wichtige Autoritäten ihres Denkens darstellten. ${ }^{49}$

Als Beleg der Vorläuferschaft des Euripides für den Pyrrhonismus wird bei Diogenes Laertios aus Euripides' Hiketiden Adrastos zitiert: Dieser möchte erklären, wie menschliche Hoffnungen, Städte und Leistungen leicht zerstört werden können, indem er mit einer rhetorischen Frage bekundet, dass man bei den Menschen nicht von einem eigenen Denken ( $\varphi \rho$ ovعĩv) sprechen könne, da sie in ihrem Denken und Handeln ganz von Zeus abhingen (Eur. Suppl. 734-736). Diesem Zitat vorangegangen war ein Archilochos-Zitat (fr. 68 West), wonach das Sinnen ( $\theta u \mu o ́ s)$ der Menschen täglich von Zeus geleitet werde. Beide Zitate zusammen werden in Anschlag gebracht für die

46 Stobaios hat etwa die Überschrift „Dass die, die Menschen sind und die Pflicht haben, gemäß der Tugend zu leben, die ihnen widerfahrenden Ereignisse edel ertragen sollen“ (Stob. 4,108, darin z. B. Eur. Bellerophontes fr. 287 Kannicht als Absatz 39 oder Eur. Hypsipyle fr. 757,925f. Kannicht als Absatz 12 aufgeführt).

47 Vgl. Testimonien im Apparat bei Nauck/Snell 1964, 685 und Kannicht 2004, 988.

48 Vgl. Warren 2015, 110.

49 Nach der Auskunft eines gewissen Philon von Athen, der als Pyrrhons Freund bezeichnet wird, habe Pyrrhon Bezug „,besonders auf Demokrit, dann auf Homer“ genommen (Diog. Laert. 9,67). Sogar für die skeptische Naturphilosophie waren Homer und Euripides wichtige Autoritäten: In einem Abschnitt über Werden und Vergehen zählte Sextus Empiricus auch Homer und Euripides zwar als Vertreter einer Zwei-Elementenlehre unter Philosophen auf, die nur von einem Urelement ausgingen (z.B. Thales, Hippasos, Anaximenes, Heraklit, Xenophanes), und solchen, die mehr als zwei Elemente annahmen (z. B. Empedokles, Anaxagoras, Demokrit, Epikur); nach Homer (Il. 7,99; 14,201) seien die beiden Erde und Wasser, nach Euripides (Antiopa fr. 182a Kannicht) Erde und Äther (Math. 10,313318). 
skeptische Grundhaltung, die Natur und das Denken des Menschen als ephemer und flüchtig anzusehen. ${ }^{50}$ Das Archilochos-Fragment kehrt auch in Sextus Empiricus' Gegen die Logiker (Math. 7,128) wieder, eingerahmt von einem Zitat aus Homer (Od. 18,136f.) und einem aus Euripides' Troerinnen (885-887). Die Zitate dort sollen allerdings die Lehre Heraklits illustrieren, dass das Kriterium der Wahrheit die Vernunft (入óyos) und nicht die Sinneswahrnehmung sei und dass die Vernunft allen Menschen gemeinsam und göttlich sei (Math. 1,127), d.h. dass sie von der göttlichen Vernunft abstammt. Während hier die menschlichen Erkenntnismöglichkeiten positiv eingeschätzt werden, werden sie dort in der Liste des Diogenes Laertios - und zwar teilweise mit Berufung auf dieselben Zitate - negativ eingeschätzt. ${ }^{51}$

Die meisten Zitationen aus Euripides bei Sextus Empiricus finden sich in seiner Schrift Gegen die Grammatiker. Das spiegelt die zeitgenössische Praxis der Grammatiker, der ersten Lehrer höherer Bildung, wider, anhand der Lektüre klassischer Dichtung die Sprach- und Denkfähigkeit der Schüler zu trainieren, die Sextus hier kritisch bespricht. Sextus argumentiert gegen die Behauptung der Grammatiker, ihr Fach habe einen lebenspraktischen Nutzen, insofern die Dichtung „viele Ausgangspunkte zur Weisheit und zum glücklichen Leben“ habe, aber der Erklärung durch die Grammatik bedürfe (Math. 1,270). Das Verhältnis von Dichtung und Philosophie stelle sich für sie nach Sextus so dar:

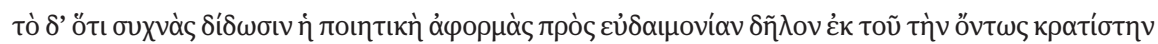

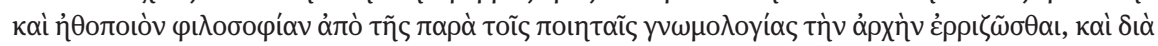

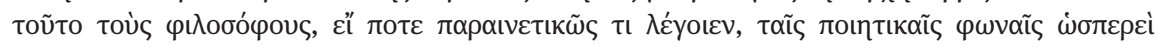

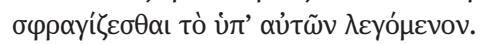

Dass die Dichtung zahlreiche Anregungen zum Glück gibt, sei daraus klar, dass die wahrhaft stärkste und charakterbildende Philosophie zu Anfang in den gnomischen Aussprüchen der Dichter verwurzelt gewesen sei und daher die Philosophen, wenn sie etwas als Paränese sagen, durch Dichterworte das von ihnen Gesagte gleichsam besiegeln. $(1,271)^{52}$

Das heißt also: 1) Die Dichtung (wie auch die sie interpretierende Grammatik) hat ihr Ziel nicht im Vergnügen oder einer theoretischen Erkenntnis, sondern im praktischen Nutzen. Dieser ist die individuelle Eudaimonia, zu deren Ausbildung es der Charakterbildung durch die Philosophie, speziell die Ethik, bedarf. Die bevorzugte philoso-

50 Vgl. Warren 2015, $114 \mathrm{f}$.

51 Vgl. Warren 2015, 115. Ähnlich konnte auch das oben genannte Zitat aus Euripides' Hiketiden nicht nur negativ wie in Diogenes Laertios' Pyrrhonismus-Kapitel gelesen werden, sondern auch positiv: Bei Plutarch (Stoic. repug. 1056B) wird es von Chrysipp zitiert, um, wie Plutarch sagt, zu zeigen, dass kein Stillstand und keine Veränderung ohne Zeus' Willen möglich seien und dass der gesamte Kosmos von einer höchsten göttlichen Ursache geleitet werde. Die Verwendung des Hiketiden-Zitats zugunsten einer skeptischen Erkenntnistheorie hat somit vermutlich eine anti-stoische Pointe (vgl. Warren 2015, 114).

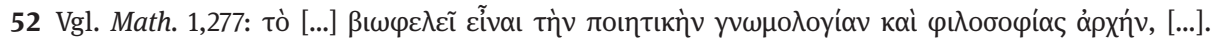


phische literarische Gattung ist daher die Paränese, die pädagogisch motivierte Ermahnung.

2) Dichtung und Philosophie werden historisch und funktional aufeinander bezogen: Beide haben dasselbe Ziel, nämlich die individualethische Charakterbildung, allerdings kommt die Dichtung in der Entwicklung der Disziplinen sowie in der Unterrichtspraxis chronologisch vor der Philosophie. ${ }^{53}$ Sie ist deshalb allerdings keine embryonale Vorform der Philosophie, sondern dieser aufgrund ihres Alters und ihrer Ursprünglichkeit in gewisser Weise an Wertigkeit sogar vorgeordnet. Daher bemühen auch zeitgenössische Philosophen immer wieder Dichterzitate, mit denen sie ihre Aussagen beglaubigen und ihnen Nachdruck verleihen. Die Zitate fungieren hier als Argument aufgrund der auctoritas der älteren Dichtung. ${ }^{54}$ Möglicherweise hat Sextus hier eine stoische Position im Blick. ${ }^{55}$ Man denke an den schon genannten Chrysipp, ${ }^{56}$ an Kleanthes, dessen bekanntestes Werk sein in Versen verfasster Zeus-Hymnos ist, ${ }^{57}$ oder etwa an das Ende von Epiktets Encheiridion. ${ }^{58}$

3) Die Philosophen sind ausdrücklich nur an den Gnomen der Dichter interessiert. Für ihre Zwecke ist daher nicht die Lektüre der gesamten Werke der Dichtung nötig, sondern es genügen Gnomologien, die bereits von den Grammatikern selbst für den Schulgebrauch angefertigt worden sind. ${ }^{59}$ Dieser Praxis folgt Sextus selbst, wenn er im Anschluss an das oben genannte Zitat Euripides-Sentenzen anführt $(1,271),{ }^{60}$ die so oder ähnlich als offenbar feststehende ,geflügelte Worte‘ auch bei anderen Autoren vorkommen ${ }^{61}$ und daher vermutlich entsprechenden Gnomologien entnommen sind.

53 Bereits die Sophisten verwiesen auf die chronologische Priorität ihrer Weisheit zur Dichtung (vgl. Plat. Prot. 316d3-7). Bereits zu Protagoras' Zeiten bestand die Unterrichtspraxis der Elementargrammatiklehrer, darin nach dem Erlernen der Schrift klassische Dichtungen mit vorwiegend moralischem, ermahnenden Inhalt zu lesen (vgl. Plat. Prot. 325e1-326a4).

54 Mehr auf die emotive Wirkung der Dichtung bezieht Blank 1998, 286 diese Stelle: „Further, the poets have expressed the same thing as philosophers, but in a manner better suited to move people to follow their precepts."

55 Vgl. Blank 1998, 281-286.

56 Vgl. oben S. 302 mit Anm. 8.

57 Kleanthes war der Meinung, dass die dichterische Form die Aufmerksamkeit des Lesers für die in ihr enthaltene Wahrheit schärfe (SVF 1,487) und dass sie für göttliche Gegenstände sogar angemessener sei als Prosa (SVF 1,486).

58 Vgl. oben S. $305 \mathrm{f}$.

$59 \mathrm{Zu}$ Gnomologien siehe oben den Beitrag von Rosa Maria Piccione in diesem Band.

60 Als Mahnung zur Tugend Eur. Temenidae fr. 734,1 Kannicht (über die Unverlierbarkeit der Tugend), zur Absage an die Geldesliebe Eur. Aeolus fr. 20 Kannicht (über die ethische Indifferenz des Reichtums) oder zur Autarkie Eur. fr. 892,1-3 Kannicht (lebensnotwendig für den Menschen sind nur Wasser und Brot).

61 Vgl. Blank 1998, 287f. zu anderen Vorkommen der Euripides-Zitate. Blank 1998, 302f. bemerkt auch, dass die hier zitierten Euripides-Fragmente (20 und 892,1-3 Kannicht) und das in Math. 1,278 im selben Kontext (nach fr. 20 Kannicht) zitierte Fragment 324 Kannicht auch in einer Passage bei Athenaios über Armut und Reichtum (4,48f., 158d4-159d10) zitiert wird, und vermutet u.a. aufgrund der weiteren in dieser Passage vorkommenden Zitate aus Chrysipp, dass auch die Athenaios-Zitate aus Chrysipps Politeia oder aus einer stoischen Anthologie von Dichterzitaten stammten. 
Sextus' detaillierte Kritik an der Behauptung der Grammatiker, die Grammatik bzw. die Dichtung sei nützlich für das Leben, stützt sich nun weiter auf epikureische Argumente gegen diese offenbar stoische Position. ${ }^{62}$ Die Argumentation geht auf der Grundlage gewisser Hypothesen vor: Zunächst wird der lebenspraktische Nutzen der Dichtung, sofern sie gnomisch und paränetisch ist, zugestanden, der Nutzen der Grammatik aber bestritten, weil die Dichtung nützlich sei, die von selbst deutlich ( $\sigma \alpha \varphi \tilde{\omega} \varsigma$ ) sei und keiner Grammatik bedürfe, andernfalls (z. B. in ausländischen oder allegorischen Geschichten) sei sie nutzlos (1,278). Anschließend wird das Argument umgedreht und zugestanden, dass die Grammatik nützlich sei, die Dichtung aber

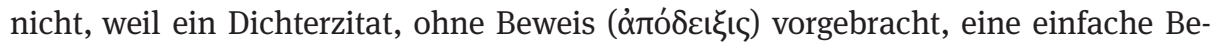

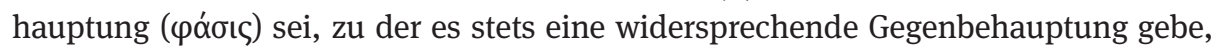
und so die Menschen zum Schlechteren verleite; daher sei für die echten Philosophen nur der Beweis und nicht das Dichterwort ein hinreichendes Überzeugungsmittel, während sich die Philosophen, die die Menge täuschen wollen, nur auf dieses stützten (1,279f.). ${ }^{63}$ Als Beispiel einander widersprechender Dichterworte führt Sextus verschiedene Euripides-Zitate an, von denen das eine den Wert des Reichtums leugnet (Eur. Aeolus fr. 20 Kannicht), die anderen diesen vehement anpreisen (Eur. Danae fr. 324,1-4 Kannicht; Phoen. 403; adesp. 464 Kannicht).

An dieser Passage ist klar zu sehen, dass Sextus (bzw. seine epikureische Quelle) Dichterzitate nicht nur im Horizont der Bildung und pädagogischen Paränese sieht, sondern klar mit einem streng philosophischen Fokus. Die Dichtung wird am Maßstab der Philosophie gemessen: Poetische Gnomen werden aufgrund ihrer allgemeinen Form als allgemeingültige Aussagen verstanden und philosophischen Behauptungen gleichgesetzt. Sie werden zu Prämissen einer philosophischen Argumentation oder zu Sätzen, die prinzipiell aus einer philosophischen Argumentation als Konklusion hervorgegangen sein können. Daher kann an sie - ähnlich wie auch an philosophische Lehrsätze - das Kriterium der Widerspruchslosigkeit angelegt werden, ohne Rücksicht auf ihre ursprüngliche dramatische Funktion und ihren ursprünglichen Kontext, aber auch ohne Rücksicht darauf, ob die inkriminierten Widersprüche der Satzaussagen nicht auch einen Nutzen haben können, etwa indem sie das Denkvermögen üben oder den Anstoß zur besseren Argumentation geben. ${ }^{64}$

Nach dieser Darlegung, wie Sextus Dichterzitate im Allgemeinen und EuripidesZitate im Besonderen für methodologische Fragen in Dienst nimmt, sei noch kurz auf seine Ethik und Güterlehre eingegangen, in der auch Euripides-Zitate eine besondere Rolle spielen. Sextus argumentiert in den Pyrrhonischen Hypothesen dafür, dass es

62 Vgl. Math. 1,299; Blank 1998, 286. 296-298. 305.

63 Dass es sich um die Wiedergabe einer fremden, nämlich epikureischen Position handelt, ist daran ersichtlich, dass Sextus selbst skeptisch ist sowohl hinsichtlich der einfachen Behauptung, da es hierzu eine widersprechende Gegenbehauptung gebe, wie auch hinsichtlich des Beweises, da dieser von Prämissen abhängt, deren Unbezweifelbarkeit bestritten werden kann (vgl. Math. 1,157. 188; Blank 1998, 297 f. 305).

64 Vgl. Plut. De aud. poet. 20C7-E2. 
nichts von Natur ( $\varphi$ v́øı) aus Gutes oder Übles gebe, sondern alles konventionell

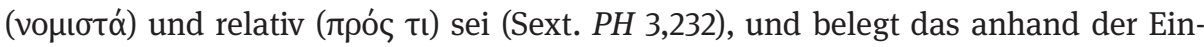
schätzung des Todes. Unter den Autoritäten, die den Tod für nicht meidenswert halten, wird als erster Euripides (fr. 638 Kannicht) aufgeführt mit der Frage, wer wisse, ob nicht das Leben Totsein sei und das Totsein als Leben unten gelte, ${ }^{65}$ gefolgt von einem Epikur-Zitat (Sent. 2), wonach der Tod uns nichts angehe, weil er die Auflösung von Seele und Körper und damit einerseits Empfindungslosigkeit, andererseits unsere (personale) Abwesenheit bedeute, und anschließend einer Heraklit-Paraphrase, dass Leben und Sterben sowohl in unserem Leben als auch im Tod seien, weil, während wir lebten, unsere Seelen tot und in uns begraben seien und, wenn wir stürben, sie wieder lebendig würden und lebten ( $P H$ 3,229f.). ${ }^{66}$

Während diese drei Zitate begriffsanalytisch-dialektisch verfahren und die implizite begriffliche Indifferenz von Leben und Tod belegen sollen, bringt der folgende Passus (3,230 - 232), wiederum eingeleitet mit einem Euripides-Zitat, die Präferenz des Todes gegenüber dem Leben zum Ausdruck. Auf das Zitat aus Euripides' Kresphontes (fr. 449,3-6 Kannicht), dass Neugeborene wegen bevorstehender Übel beweint, Tote hingegen mit frohem Gesang aus dem Haus geleitet werden sollten, folgt das berühmte Theognis-Fragment (425-428), dass nicht geboren zu sein das Beste sei und, wenn man doch geboren sei, möglichst schnell zu sterben. Und gleichsam wie eine historische Bestätigung der Euripides-Verse wird aus Herodot von Kleobis und Biton erzählt, deren früher Tod als göttliches Zeichen angesehen wird, dass es besser sei tot als lebendig zu sein (Hdt. 1,31), und von einigen Thrakern, die Neugeborene angesichts der ihnen bevorstehenden Übel beweinten $(5,4){ }^{67}$

Die meisten der hier aufgeführten Zitate - bis auf das Heraklit-Zitat - finden sich auch bei Stobaios (4,120), so dass man schließen kann, dass Sextus wie Stobaios eine Sammlung von Zitaten benutzten, die unter einer ähnlichen Überschrift wie bei

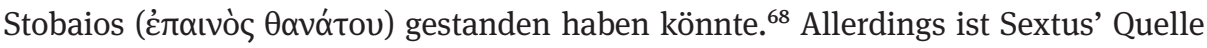

65 Ähnlich auch Eur. Phrixus I oder II fr. 833 Kannicht. Bei Diogenes Laertios findet sich dasselbe Zitat im Abschnitt über die Vorläufer des Pyrrhonismus, allerdings als Beispiel für fallible menschliche Meinungen im Gegensatz zur göttlichen Wahrheit (Diog. Laert. 9,73).

66 Vgl. DK 22 B 88 (= Plut. Cons. ad Apoll. 106E). Sextus paraphrasiert das Heraklit-Fragment unter platonischem Blickwinkel, denn nach Plutarch sagt Heraklit nur, dass in uns Leben und Tot-Sein, Wach- oder Schlafend-Sein, Jung oder Alt dasselbe seien, weil das eine in das andere umschlage und umgekehrt.

67 Ausgelassen ist bei Sextus die Fortsetzung bei Herodot, dass sie den Verstorbenen freudig bestatteten, weil er von allen Leiden befreit und nun in völliger Glückseligkeit sei. Bei Clemens von Alexandria (Strom. 3,3,15,1-16,1) ist Theogn. 425-27 gefolgt von Eur. Cresphontes fr. 449,3-6 Polyidus 638 Kannicht sowie Hdt. 1,31 (vgl. Kannicht 2004, 486).

68 Eur. Polyidus fr. 638 Kannicht = Stob. 4,120,38 (zwei zusätzliche Verse, die beiden überlieferten Verse variieren); DL 10,125 = Stob. 4,118,29 (das Zitat steht hier in einer Passage mit der Überschrift „Über den Tod und dass er unvermeidbar ist“); Eur. Cresphontes fr. 449,3- 6 Kannicht = Stob. 4,120,42; Theogn. 425-428 = Stob. 4,120,30 (die beiden letzten Worte bieten eine Textvariante); Hdt. 1,41 = Stob. 4,120,43; Hdt. 5,4 = Stob. 4,120,53; vgl. Pappenheim 1881, 262. Das Zitat findet sich auch bei den 
offenbar eine epikureische Sammlung, da sie sich auf die Zitate Epikurs und des Euripides konzentrierte, dazu das Theognis-Fragment, das bereits bei Epikur selbst eine entscheidende Rolle gespielt hat (Epicur. Ep. Men. 126). Zum Vergleich: Stobaios führte unter der genannten Überschrift „Lob des Todes“ auch Zitate anderer Dramatiker und Philosophen auf. ${ }^{69}$ Außerdem gibt es vor diesem Kapitel eine Sammlung von

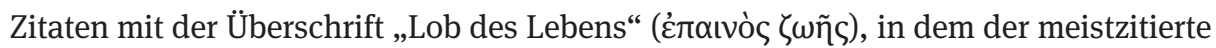
Tragiker auch Euripides ist. ${ }^{70}$ Solche Zitate dürften in Sextus' epikureischer Quelle fehlen, weil sie der epikureischen These von der Bedeutungslosigkeit des Todes für das Leben entgegenstehen. Sextus hingegen ließ die allseits akzeptierte Ansicht vom Wert des Lebens beiseite und konzentrierte sich argumentativ ganz auf das ,Lob des Todes', um der weniger plausiblen Ansicht philosophische Autoritäten zur Unterstützung an die Seite zu stellen und damit das übergeordnete skeptische Ziel der

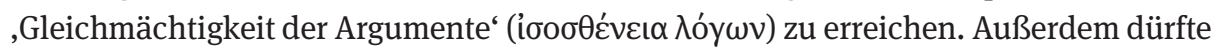
das eher seiner eigenen Ansicht der Indifferenz von Tod und Leben entsprochen haben. ${ }^{71}$

\section{Neuplatonismus}

Der Neuplatonismus, der die dominierende philosophische Schule der ausgehenden Kaiserzeit von der Mitte des 3. bis zur Mitte des 6. Jh. n. Chr. darstellte, weist naturgemäß eine große Bandbreite von philosophischen Meinungen auf, trotz einiger gemeinsamer Grundannahmen, etwa dem gemeinsamen Rückbezug auf Platons Philosophie als die Wahrheit. Die Haltung zur Dichtung und damit zu Euripides unterliegt den verschiedenen individuellen Interessen der einzelnen Autoren und ihrer jeweiligen Positionierung als Philosoph gegenüber dem der Philosophenschule vorausliegenden Grammatik- und Rhetorikunterricht. Im Folgenden werden nur die Neuplatoniker behandelt, deren Euripides-Zitationen besonders zahlreich und/oder besonders signifikant für den neuplatonischen Gebrauch des Euripides sind. ${ }^{72}$

\footnotetext{
Neuplatonikern Iamblich (Protr. 17, 84,7-9 Pistelli) und Olympiodor (In Gorg. 29,3, 153,13-15 Westerink), vgl. hierzu unten S. 327.

69 An Dramatikern z.B. Aischylos, Sophokles, Menander, wenn auch mit einem zahlenmäßigen Übergewicht des Euripides, und an Philosophen z.B. Anaxagoras, Demokrit, Platon - Apologie, Phaidon und der ps.-platonische Axiochos - sowie Themistios (Stob. 4,120,20-55).

70 Acht von 19 Zitaten, u. a. aus dem Orestes, der Iphigenie in Aulis und dem Hippolytos.

71 Nach Stob. 4,53,28 (= T 19 Deleva Caizzi) habe Pyrrhon zwischen Leben und Totsein „keinen Un-

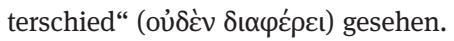

72 Nicht eigens behandelt wird etwa Synesios von Kyrene, obwohl sich bei ihm, vor allem in seinen Briefen, recht viele Euripides-Zitate finden. Sein Zitatgebrauch entspricht der gängigen rhetorischen Praxis, was seiner eigenen Verbindung von Philosophie und Rhetorik entspricht (vgl. Syn. Dio 5, 246,21-247,1 Terzaghi; Ep. 1,1-3). So finden sich bei ihm vor allem Anspielungen auf geflügelte Worte (z. B. Eur. Or. 397 in Syn. Insomn. 1, 144,1f. Terzaghi; Ba. 881. 901 in Ep. 138, 241,17f. Terzaghi) und Schmuckzitate (Or. 259 in Ep. 12, 32,13f. Terzaghi; Or. 604 in Ep. 105, 186,15 Terzaghi; Ba. 485 in Ep. 105,
} 


\subsection{Plotin}

Plotin hatte kein besonderes Interesse an der Dichtung, zeigte aber Anzeichen einer symbolischen Deutung der dichterischen Mythen, wie sie dann vor allem bei den späteren Neuplatonikern entwickelt worden ist. ${ }^{73}$ So scheint bei ihm bereits eine Zweiteilung der Dichtung in eine archaische, „vom unvergänglichen Logos erfüllte“ Dichtung, zu der Homer und die später entstandenen, aber als archaisch geltenden orphischen Gedichte zählten, und eine Klasse von kanonischen Gedichten wie Theognis und die Tragiker, die nicht mit dem ewigen philosophischen Logos übereinstimmten, vorgezeichnet zu sein, die dann bei Proklos weiter ausgeführt worden ist. ${ }^{74}$ Entsprechend ist auch bei Plotin Homer der am meisten zitierte Dichter: Auf ihn finden sich, ohne Nennung des Zitatautors, 28 Anspielungen. ${ }^{75}$ Von Euripides finden sich bei ihm etwa ein halbes Dutzend namentlich nicht gekennzeichnete Zitate gegenüber dreien aus Aischylos und dreien aus Sophokles; ${ }^{76}$ von den vollständig erhaltenen Tragödien gibt es nur ein Zitat, nämlich aus den Troerinnen: „Auf lautloser Bahn dem Recht gemäß“ (Tro. 887 f.) führe Zeus alle Dinge der Menschen, sagt dort die Troerkönigin Hekabe erfreut, deren Familie und Stadt nach dem Trojanischen Krieg zerstört und sie selbst versklavt werden soll, nachdem Menelaos angekündigt hat, dass er Helena töten lassen werde; freilich ahnt sie, dass diese Ankündigung nicht durchgeführt werden wird. ${ }^{77}$ In der Anrede an Zeus kombiniert Hekabe traditionelle religiöse und zeitgenössische philosophische Vorstellungen, u. a. die „Notwendigkeit

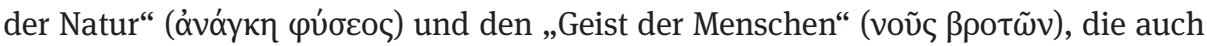
als Neuerung wahrgenommen werden $(884-886.889){ }^{78}$

Dass Plotin die Troerinnen des Euripides kannte, ist nicht sehr wahrscheinlich. Es handelt sich bei dem Zitat um ein geflügeltes Wort, das häufig auch von anderen

184,15f. Terzaghi; fr. 723 Kannicht in Ep. 101, 171,3 Terzaghi). Kaum oder gar keine Euripides-Zitate finden sich hingegen bei Iamblich, Hermeias, den Philosophen der Schule des Ammonios Hermeiou in Alexandria (mit Ausnahme des Olympiodor, was daran liegen dürfte, dass von ihm als einzigem aus dieser Schule Kommentare zu Werken Platons erhalten sind) sowie Damaskios.

73 Lamberton 1986, 83f. Umfassend zum Verhältnis Plotins zu Mythos und Dichtung Cilento 1960. 74 Dörrie (in Cilento 1960, 316f.). Zur Dreiteilung der Dichtung bei Proklos vgl. unten S. $320 \mathrm{f}$.

75 Vgl. Cilento 1960, 277-290; Lamberton 1986, 90 -107. Eine Übersicht über alle Dichterzitate findet sich bei Cilento 1960, 271-305.

76 Vgl. Cilento 1960, 298-302. Cilento führt für Euripides - außer dem im Folgenden besprochenen Zitat - auch Melanippe (I) Sapiens fr. 486 Kannicht an, das zweimal (1,6,4,11f. und 6,6, 6,39f.) verwendet wird (das Zitat „Antlitz der Gerechtigkeit, weder Abendstern noch Morgenstern so wunderbar“ [bei Plotin statt „wunderbar“ „schön“] wird zum Ausdruck der geistig erfassbaren Idee der Gerechtigkeit benutzt), dazu zwei weitere Zitate (u.a. Eur. fr. 944 Kannicht in Plot. 4,4,27,15f.). Nicht bei Cilento verzeichnet ist das unten besprochene Zitat Eur. fr. 1018 Kannicht (in Plot. 6,5,1,1-4). Man müsste also von mindestens fünf bzw. sechs (weil ein Zitat zweimal verwendet wird) Zitaten sprechen.

77 Vgl. Eur. Tro. 1036-1059.

78 Vgl. z. St. Biehl 1989, 334-337. 
Autoren für das Walten der Götter zitiert wurde. ${ }^{79}$ Dennoch ist es nicht unwahrscheinlich, dass Plotin das Leid der Hauptperson Hekabe sowie ihr Vertrauen auf die ausgleichende göttliche Gerechtigkeit, die dann jedoch enttäuscht wird, kannte und im Sinne des bereits bei den Stoikern praktizierten Verfahrens, den Dichter bzw. dessen Figuren, deren Aussagen und Handlungen zu korrigieren, interpretierte. ${ }^{80}$ Das Zitat lautet nämlich vollständig: Alles gehe im All seinen Gang ,auf lautloser Bahn dem Rechte gemäß“; der Schlechte wisse davon nichts und werde, ohne es zu merken, dahin gelenkt, wohin er gehöre, während der Gute darum wisse und den ihm angestammten Platz kenne und guter Hoffnung auf ein Leben bei den Göttern sei (Plot. 4,4,45,27-33). Das Zitat ist also nicht nur auf die kosmologische Ordnung, sondern auch auf das Verhalten des Menschen zu seinem Schicksal bezogen. Vor dem Hintergrund der euripideischen Tragödie wäre dies eine Korrektur der Hekabe unter platonischen Vorzeichen: Sie hätte Recht damit, die Notwendigkeit des Kosmos anzuerkennen. Wirklich moralisch gut im Sinne des plotinischen Weisen wäre sie allerdings erst dann, wenn sie ihren vorherbestimmten Platz in der Welt akzeptierte, sich also in ihr Schicksal als Kriegsgefangene des Odysseus fügte und mit ihm von Troja nach Ithaka ginge und darüber keine Klage wie in der Tragödie führte, sondern vielmehr ihre Hoffnung auf die wahre Heimat der Seele bei den Göttern setzte, was der Hoffnung des Sokrates auf ein Leben nach dem Tod entspricht (Plat. Phd. 63b5-c7).

Unter Plotins wenigen Euripides-Zitationen findet sich auch das sehr bekannte

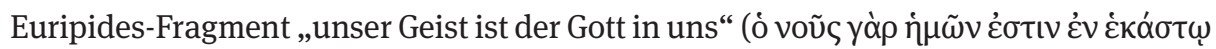
$\theta \varepsilon$ ós, Eur. fr. 1018 Kannicht), das offenbar häufig zitiert wurde. ${ }^{81}$ Plotin beginnt seine Erörterung über die Einheit und Totalität des geistig Seienden in Anlehnung an dieses Zitat, das er allerdings einer „allgemein verbreiteten Vorstellung“ (кoเvท̀ हैvvoı $\alpha$ ) zuschreibt und im Hinblick auf sein Darlegungsziel anpasst: Alle würden behaupten, dass der Gott, der in jedem einzelnen von uns sei, ein und derselbe sei (Plot. 6,5,1,1-4). Diese These von der Einheit des Geistes in der ganzen Vielheit aller seiner Instanzen geht zurück auf Plotins Interpretation des Parmenides. ${ }^{82}$ Sie nimmt das Zitat, das ursprünglich vermutlich nur die individualethische, relativistische Aussage hatte, dass jeder Mensch seines eigenen Kopfs sei, dem er als seinem Gott folge, nicht nur wie andere Philosophen, die das Zitat benutzen, aufgrund der Ausdrücke voũ s und

79 Z. B. Clem. Al. Protr. 2,25,3; Sext. Math. 1,288; 7,128; Plut. Quaest. Plat.1007C3f.; An. procr. 1026B8f.; Ps.-Iustin. Mon. 5; Procl. Theol. Plat. 1,13, 59,14-20 Saffrey-Westerink; 1,16, 77,8f.; 1,18, 87,17-21; 6,23, 101,19-22; In Tim. 1, 398,16-18; Syn. Regn. 17, 40,1-6 Terzaghi.

80 Auch Longo 2016, die die Verwendung von Eur. Tro. $887 \mathrm{f}$. bei Plutarch, Plotin und Proklos untersucht, betont, dass Plotin den ursprünglich leiderfüllten Kontext des Tragödienzitats kannte und ihn im Sinne der platonischen Theodizee zu einer optimistischen Aussage veränderte.

81 U.a. Theon Progymn. 103,5-7 Spengel; Marc. Aur. 12,26,2; Nemes. Emes. Nat. hom. 43, 127,22f. Morani (Euripides und Menander zugewiesen); Plut. Quaest. Plat. 999D (nur Menander zugewiesen); Iambl. Protr. 8, 48,15-17 Pistelli (= Arist. Protr. fr. 61, 72,7-9 Rose) (Hermotimos oder Anaxagoras zugewiesen); zu weiteren Testimonien siehe den Apparat bei Nauck/Snell 1964, 685 und Kannicht 2004, 988.

82 Vgl. Tornau 1998, 323. 


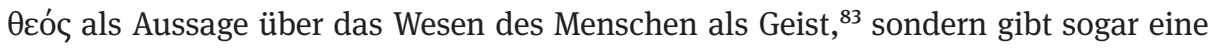
metaphysische Begründung für die Zugehörigkeit jedes einzelnen menschlichen Geistes zum transzendenten göttlichen Geist, was die Aussage - vermutlich entgegen ihrer ursprünglichen Intention - gerade absolut und nicht-relativistisch verstehen würde. Die Wichtigkeit dieses Zitats für Plotin bezeugen die von Porphyrios überlieferten letzten Worte des sterbenden Plotin, dass er „versuchen will, das Göttliche in

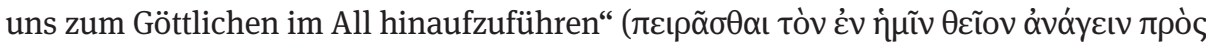

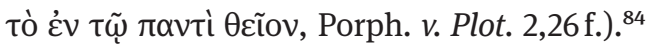

\subsection{Porphyrios}

Einen wesentlichen Beitrag zur Entwicklung der neuplatonischen Dichterexegese hat Plotins Schüler Porphyrios geleistet. Insbesondere die Homer-Interpretation stand im Fokus seiner philologischen Beschäftigung. Erhalten sind hiervon seine Homerischen Fragen, in denen er nach dem Prinzip Aristarchs, Homer aus Homer zu erklären, vorging, während er die Nymphengrotte in der Odyssee (De antro nympharum zu Hom. Od.13,102-112) in der von Numenios geprägten allegorischen Interpretationsmethode auslegte. ${ }^{85}$ Dem gegenüber fällt das Interesse an den Tragikern eher ab. Zahlreicher als bei Plotin, aber dafür philosophisch weniger gehaltvoll sind die Euripides-Zitate bei ihm, die er überdies auch nicht philosophisch interpretiert. Vielmehr verwendet er, der auch Schüler des Philologen und Philosophen Longin war, sie gemäß der in der Grammatiker- bzw. Rhetorenschule geübten Praxis: Die Zitate fungieren bei ihm als literarische Bestätigung eines philosophischen Sprachgebrauchs ${ }^{86}$ oder einer allge-

83 Eine wichtige „Zwischenstation“ für ein solches philosophisches Verständnis der Stelle ist gewiss durch den Einfluss des Aristoteles gegeben, der „unseren Geist“ als „Gott“ (Protr. fr. 61, 72,7-9 Rose) bzw. den Geist als „etwas Göttliches in uns“ (Eth. Nic. 10,7, 1177b28) bezeichnete.

84 Zitiert nach der editio maior: Paul Henry u. Henri-R. Schwyzer (Hgg.), Plotini Opera, Bd. 1 (Porphyrii Vita Plotini, Enn. I-III), Paris/Brüssel/Leiden 1951. Zu den überlieferten Varianten vgl. die editio minor: Paul Henry u. H.-R. Schwyzer (Hgg.), Plotini Opera, Bd. 1 (Porphyrii Vita Plotini, Enn. I-III), Oxford 1964 und in den „Addenda et corrigenda ad textum et apparatum lectionum“ in der editio minor, Bd. 3 (Enn. VI), Oxford 1982, 304. Überliefert sind auch die Varianten tòv ćv úfĩv $\theta \varepsilon \tilde{o}$ v bzw. $\theta \varepsilon o ́ v$, und zusammen mit der Konjektur $\pi \varepsilon\llcorner\rho \tilde{\alpha} \sigma \theta \varepsilon$ statt des einhellig überlieferten Infinitiv würden die letzten Worte Plotins eine Mahnung an die Schüler bedeuten (von Schwyzer ist im Museum Helveticum 33, 1976, 95 auch die

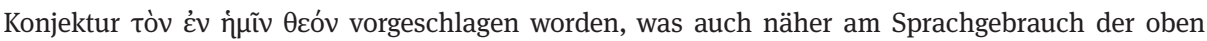
besprochenen Passage Plot. 6,5,1,1-4 und ihrer Euripides-Reminiszenz wäre). Zum Problem dieser Stelle vgl. J. Pépin, „La dernière parole de Plotin (VP 2.23.27)“, in: Brisson u. a. 1992, 355-383.

85 Vgl. hierzu ausführlicher Lamberton 1986, 108-133 (bes. 109).

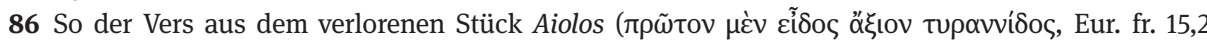

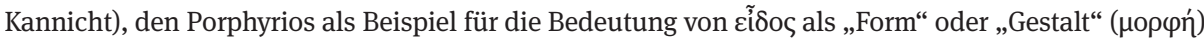
(Porph. Isag. 3,22-4,1; hiervon ausgehend auch Elias, In Isag. 61,12-14 Busse; David, In Isag. 143,22f. Busse, der den Vers den Phönissen zuweist; Ps.-Elias, In Isag. 32,7 Westerink). 
mein verbreiteten Ansicht, ${ }^{87}$ als Beispiel für bestimmte grammatikalische Phänomene ${ }^{88}$ oder als rhetorischer Schmuck. ${ }^{89}$

Eine argumentative Funktion hat das längere Zitat aus der Parodos der verlorenen euripideischen Tragödie Die Kreter, das Porphyrios in De abstinentia unter namentlicher Nennung des Autors ausführlich anführt. Darin stellt sich der Chor aus sogenannten „Propheten des Zeus“, vermutlich Priesterinnen des kretischen Zeusorakels, dem Kreterkönig Minos als „Myste“ des idäischen Zeus, des Dionysos und der Kybele vor und behauptet für sich eine zölibatäre und vegetarische Lebensweise (Eur. fr. 472 Kannicht; Porph. Abst. 4,19). ${ }^{90}$ Das Zitat steht im größeren Kontext einer Widerlegung der Ansicht, dass in der Vergangenheit niemand den Vegetarismus praktiziert habe (Abst. 4,1), die in der Folge durch viele historische Beispiele, angefangen von den Griechen über die Ägypter, die Juden, die Syrer, die Perser, die Inder bis hin eben zu den Kretern, entkräftet wird. Angeführt werden Belege aus der Historiographie, Geographie und Philosophie, und in der Reihe von Dichterzitaten ${ }^{91}$ stellt das Zitat aus Euripides' Kretern das längste und gleichsam den Abschluss der ethnologischen Untersuchung dar, bevor Porphyrios einige philosophische Reflexionen über Reinheit anstellt. Dass das Zitat nicht nur aufgrund seiner sachlichen Information, sondern auch aufgrund seiner rhetorischen Wirkung ausgesucht wurde, sieht man auch daran, dass der Beleg für den behaupteten Vegetarismus bei den Kretern lediglich in den letzten beiden Versen des insgesamt 19 Verse umfassenden Zitats zu finden ist.

\subsection{Proklos}

Proklos ist der erste Neuplatoniker, der versuchte, die Dichterlektüre philosophisch zu begründen, und der damit den späteren Neuplatonismus nachhaltig prägte. Er hat in dem Bemühen, alle Quellen der Weisheit in einem Denksystem zu vereinen - dazu zählen für ihn neben der platonischen Philosophie die chaldäische Orakelphilosophie

87 Über die Schlechtigkeit von Frauen werden in der Art eines Gnomologions Zitate des Euripides (fr. 494,27-29 Kannicht; Med. 231) neben Zitaten des Hesiod (Op. 702f.), des Simonides (fr. 6 West) und des Tragikers Theodektes (F1a Snell) aufgeführt (Porph. fr. 409F Smith).

88 Z. B. Eur. Hipp. 386 für die Dualform (Porph. Quaest. Hom. in Il. 1,104, 18,13 MacPhail) oder Or. 25 (neben Aristophanes und Aischylos) für eine Wortbedeutung (Quaest. Hom. in Il. 14,200, 212,30-35 MacPhail).

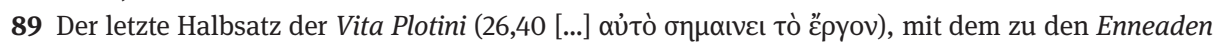

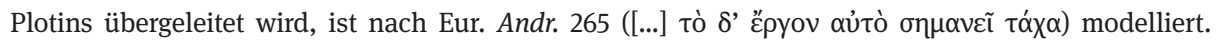
90 Zur Analyse dieses Zitats vgl. Patillon/Segonds 1995, XLIII-XLVI. Das zweite Euripides-Zitat in De abstinentia - Eur. fr. 1004 Kannicht in Porph. Abst. 3,25 - ist innerhalb eines Theophrast-Fragments

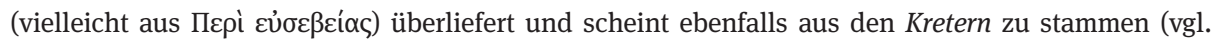
Kannicht 2004, 979).

91 Andere stammen von Hesiod und Menander: Hes. Op. 116-119 in Porph. Abst. 4,2; Men. fr. 631 Kassel-Austin in Porph. Abst. 4,15. 
und der Orphismus - ${ }^{92}$ auch Homer als Quelle der Weisheit angesehen und in das neuplatonische Denkgebäude zu integrieren versucht. Gegen die bekannte platonische Kritik im zweiten und dritten Buch der Politeia an Homer und seiner Darstellung von Göttern und Heroen, nach der dieser als Jugenderzieher nur von eingeschränktem Nutzen sei (Rep.10, 606e-607a), ${ }^{93}$ verteidigt er den Dichter und versucht plausibel zu machen, dass sowohl Homer als auch Platon „über die göttlichen Dinge dasselbe lehren“ und „Exegeten derselben Wahrheit über die Dinge“ sind, wobei Platons Lehre über die Dichtung und über Homer die „einzige, unwiderlegbare Wahrheit“ sei (In Rep. 1, 71,10-17). ${ }^{94}$ Proklos bringt dazu eine Zweiteilung von Mythen in „erzieheri-

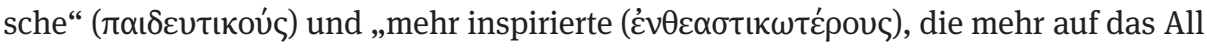
blicken als auf die Verfassung der Zuhörer“ (76,24-27), ins Spiel und behauptet, dass Homers Mythen nicht der Erziehung dienten und nicht an die Jugend gerichtet seien, sondern „der Natur des Ganzen und der Ordnung der Dinge folgen“ und „die dazu Befähigten zur Schau der göttlichen Dinge hinaufführen“ sollten (77,4-12). Damit

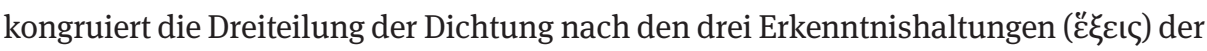
Seele: die Seele, die ihren Geist (voũc) zu den Göttern und zum Einen hin transzendiert; die Seele, die vom göttlichen Leben zu sich selbst zurückkehrt und Geist (voũৎ)

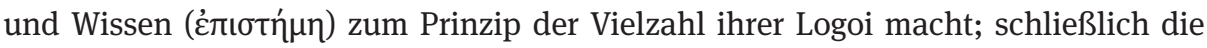

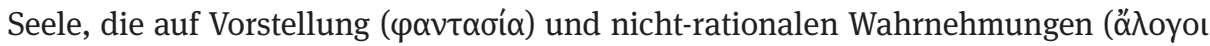

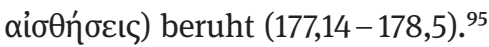

Die erste, vollkommene Art der Dichtung führt zur Erleuchtung und Einheit, ist in Anspielung auf den Phaidros (244a-245a) - ein „Wahnsinn, stärker als Beson-

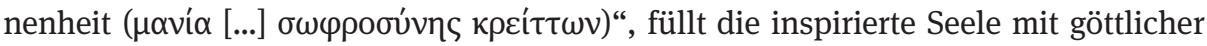
Symmetrie und ordnet noch deren geringste Tätigkeiten mit Metrum und Rhythmus (178,6-179,3); ${ }^{96}$ zu dieser Art der inspirierten Dichtung zählen Homer, Hesiod und Orpheus (72,2-5; 50,2ff.; 201,14-17). Die mittlere Art der Dichtung „erkennt das Wesen der Dinge, liebt die Schau schöner und guter Werke und Reden, führt jeden Gegenstand zu einem metrischen und rhythmischen Ausdruck“ und ist „voll von Ermahnung und guten Ratschlägen und intellektiver Mäßigung“ (179,3-15). ${ }^{97} \mathrm{Zu}$ dieser

92 Vgl. Lewy 2011, 481-485.

93 Kritisiert wird besonders die Theologie, z. B. die Götterkämpfe, die mit der Vorstellung, dass die Götter die Ursache des Guten und selber gut seien, nicht übereinstimmt (Rep. 2, 378d-380c). Kritisiert wird außerdem, dass die homerischen Helden jammern und klagen oder lachend dargestellt werden, was dem heroischen Ideal widersprechen würde, das die Wächter verfolgen sollten (3, 386a-389a). 94 Nach Sheppard 1980, 39-103 geht Proklos' Harmonisierung von Homer und Platon auf seinen Lehrer Syrian zurück.

95 Zur Dreiteilung der Dichtung bei Proklos vgl. Sheppard 1980, 162-202; Lamberton 1986, 188-195; Bernard 1990, 35-50; Kuisma 1996, 122-130; Marzillo 2010, XXII-XXVI und Lamberton 2012, XVIIXXVI. Nach Sheppard 1980, 95-103 gab es vor Proklos nur den Unterschied von inspirierter und uninspirierter Dichtung, dem dieser die Differenzierung von didaktischer und mimetischer Dichtung hinzugefügt habe.

96 Vgl. auch In Rep. 1, 182,21-186,21.

97 Vgl. In Rep. 1, 186,22-188,27. 
Art der didaktisch-paränetischen Dichtung gehörten sehr viele Werke guter Dichter (179,9 f.); Proklos nennt - ausgehend von Platons Nomoi (1, 630a-b) - Theognis, aber auch Tyrtaios (186,29-187,24). Die letzte Art der Dichtung ist die „mimetische“ ( $\left.\mu \mu \eta \tau \iota \eta^{\prime}\right)$ Dichtung, die „Meinungen und Vorstellungen zusammenmischt“, „mal nur

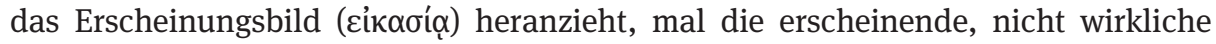

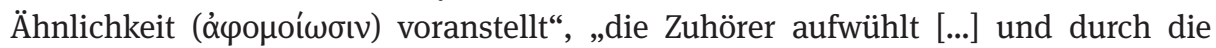
Wechsel der Harmonien und die Buntheit der Rhythmen ihre Seelenverfassung mit-

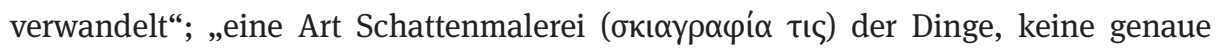

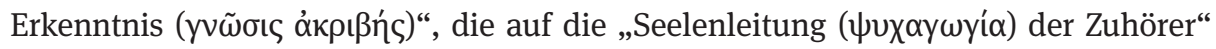

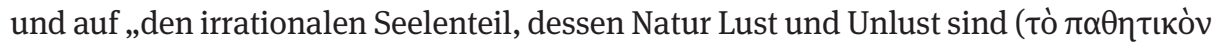

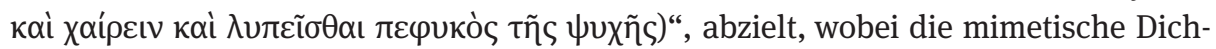

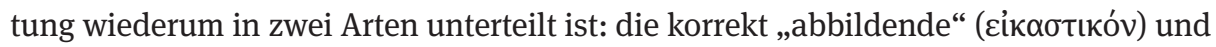

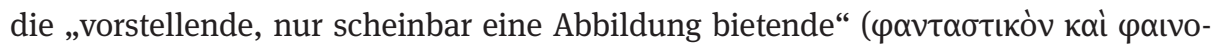

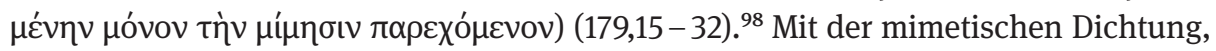
insbesondere mit deren letzter Unterart, sind in Übereinstimmung mit Platons Politeia (3, 394d-397e; 10, 595a-597e) besonders die Komödie und Tragödie gemeint (In Rep.1, 49,13-51,25; 195,21-196,9; 201,14-17). Während einzig bei der inspirierten Dichtung die neuplatonische Dichterallegorese angewandt werden kann, da diese nicht ohne philosophisch-theologische Vorbildung möglich ist, sind die niedrigeren Dichtungsarten, insbesondere die didaktische Dichtung, für junge Leute geeignet, die noch der Erziehung und moralischer Vorbilder bedürfen. ${ }^{99}$

Diese knappe Skizze der proklischen Dichtungstheorie erklärt den Befund für die Zitatpraxis recht gut: Entsprechend der Hochschätzung des Epos und der Geringschätzung der Tragödie treten die Tragödien-Zitate gegenüber den Epiker-Zitaten, vor allem Homers und auch Hesiods, weit zurück. Aber auch hier sind die Euripides-Zitate am beliebtesten; die Komödie und Sophokles werden gar nicht zitiert, Aischylos und ein unbekannter Tragiker nur jeweils einmal. ${ }^{100}$ Außerdem scheint Proklos Euripides an einer Stelle, wenn auch ungenannt, zu den „von Apollon inspirierten Dichtern“ zu zählen, wenn er auf diese die Synonymie von Helios und Apollon als Beispiel für die Einheit von hyperkosmischen Göttern mit ihren intellektiven Ursachen zurückführt (Theol. Plat. 6,12, 59,19-25 Saffrey-Westerink), ${ }^{101}$ und mit diesen Dichtern neben Ho-

98 Vgl. In Rep. 1, 188,28-191,25. Eigentlich ergibt sich also nach Proklos eine platonische Vierteilung

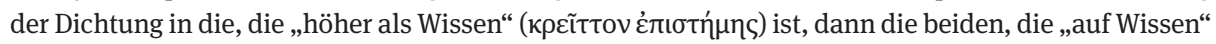

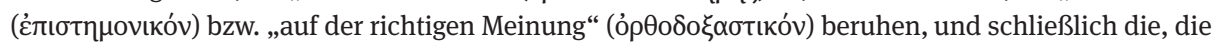

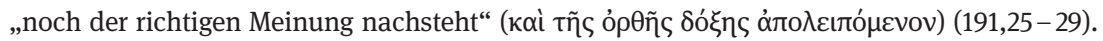

99 Vgl. In Rep. 1, 76,24-77,4; Sheppard 1980, 163; Kuisma 103-106; Marzillo 2010, XXIV. Zur neuplatonischen Dichterallegorese siehe Coulter 1976, 32-72. 102; Sheppard 1980, 145-161; Bernard 1990, 22-50; Kuisma 1996, 46-68. 77 f.; Marzillo 2010, IX-XXI.

100 Aesch. fr. 387 Radt in Procl. Theol. Plat. 3,7, 30,8 Saffrey-Westerink; TrGF 2, adesp. F 433 KannichtSnell in Procl. In Tim. 1, 338,14f.

101 Ein anderes Beispiel für theologisches Wissen ist der Orakelspruch des Apollon an Laios aus den Phönissen, in dem Laios die Zeugung von Kindern verboten wird (Phoen. 18) und den Proklos als Beispiel für die göttliche Pronoia zitiert, da der Gott zwar im Voraus wisse, dass Laios nicht gehorchen 
mer auch Euripides gemeint sein könnte; ${ }^{102}$ an einer anderen Stelle zählt er vielleicht zu den „Theologen“. ${ }^{103}$ Eine solche Hochschätzung des Euripides als Quelle theologischen Wissens scheint der allgemeinen platonischen Geringschätzung der Tragödie zu widersprechen. ${ }^{104}$ Doch hat Proklos mit seinem integrativen Modell verschiedener Arten von Dichtung eine Möglichkeit vorgegeben, Euripides als exzeptionellen Tragödienautor zu nobilitieren. Platons These, Homer sei der „Anführer“ der Tragödie (Rep.10, 598d8), deutet Proklos so, dass die Tragödiendichter Homer, der für ihn nicht nur die inspirierte, sondern auch die didaktische und die mimetische Form der Dichtung aufweist (In Rep. 1, 192,4-195,21), nacheiferten, seine Dichtung allerdings parzellierten, indem sie das bei Homer „abbildend Gesagte in der Art der Vorstellung

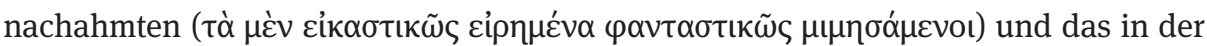

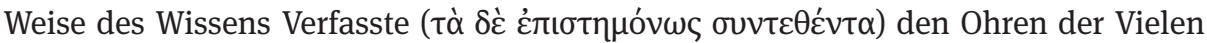
anpassten“ (196,4-9). Das heißt, dass ein Tragödiendichter durchaus höheres, auch theologisches Wissen, das für die zweite Art der Dichtung grundlegend ist, besessen haben kann und daher als Quelle höheren Wissens zur Begründung philosophischer oder theologischer Aussagen herangezogen werden darf.

Die Eigenschaft der Tragödie, Wissen der großen Menge in einer ihr angepassten Weise mitzuteilen, prädestiniert sie gerade für den philosophischen Anfängerunterricht. Namentlich genannt wird Euripides in Proklos' Kommentar zum Großen Alkibiades. Dieser Dialog wurde im Curriculum der in der Schule behandelten PlatonDialoge als erster behandelt. ${ }^{105}$ Das erklärt, warum im Vergleich zu anderen Werken des Proklos in diesem Kommentar recht viele Zitate von nicht-philosophischen Pro-

werde, aber aus Güte mit dem Orakel die Möglichkeit zu einer besseren Lebenswahl eröffne, die Laios allerdings aufgrund seiner eigenen Unvernunft nicht nutze (Procl. In Alc. 91,10-15). Dieses Beispiel dient Proklos zur Erklärung der Pronoia des Sokrates im Hinblick auf Alkibiades, der sich dieser

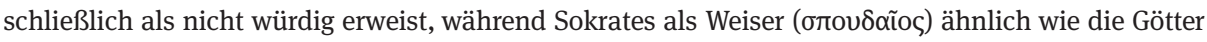
oder die Sonne ihre gute Gabe allen ungeteilt zukommen lassen möchte (88,13-16; 90,19-92,2).

102 Vgl. Hom. h. Ap. 371-374; Eur. fr. 781,11-13 Kannicht; außerdem Call. fr. 273 und 302 Pfeiffer; Saffrey-Westerink 1997, 59.

103 Die „Theologen“ werden neben Sokrates (Plat. Tht. 149b9f.) für das Epitheton $\Lambda$ oxía („Wöchnerin“) der Artemis angeführt (Theol. Plat. 6,22, 98,11f. Saffrey-Westerink), welches in der Literatur sonst bei Euripides (IT 1097; Suppl. 958) bezeugt ist.

104 Eine Kritik an Euripides, die allerdings nicht auf die platonische Geringschätzung der Tragödie zurückgeht, sondern der in den Grammatikerschulen üblichen Sprachkritik folgt (vgl. hierzu oben der Beitrag von Stefano Valente S. 143-147), übt Proklos an der Etymologie des Namens „Meleagros“, den

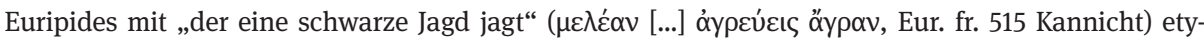
mologisiert; gemeint ist, dass er seinen Onkel getötet hatte, weil seine Geliebte Atalanta entehrt worden war (Ov. Met. 8,433ff.). Schlecht sei diese Etymologie deshalb, weil eine Etymologie sich an den Tatsachen $\mathrm{zu}$ orientieren habe und kein Vater seinem Kind einen Namen mit schlechter Vorbedeutung

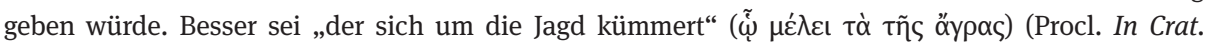
39,19-40,1 Pasquali).

105 Vgl. Procl. In Alc. 11,1-17; Anon. Prol. Plat. 26,18-20 Westerink; Festugière 1969, 285-287. Zum Iamblich-Curriculum vgl. Anon. Prol. Plat. 26,14-34 Westerink; Westerink/Trouillard 1990, LXVIIILXXIII; O’Meara 2003, $63 \mathrm{f}$. 
saautoren und Dichtern vorkommen. Das von Sokrates benutzte Euripides-Zitat aus dem Hippolytos dient dazu, Proklos' allgemeine Bemerkungen über den richtigen Gebrauch von Dichtung auf platonischer Grundlage näher zu erläutern: Sokrates hatte ja das Zitat, wonach Phaidra den Namen ihres Geliebten nicht aussprechen möchte und der Amme, die dies tut, erwidert, dass sie den Namen von sich selbst und nicht von ihr gehört habe (Hipp. 352), dazu benutzt, um im dialektischen Frage-AntwortVerfahren die Rollenverteilung zu klären und den Antwortenden, nicht den Fragenden als den Behauptenden zu kennzeichnen (Plat. Alc. 1, 113b8-c7). In der Kommentierung dieser Stelle (Procl. In Alc. 291,15-292,13) ${ }^{106}$ stellt Proklos als Leistung des Zitats

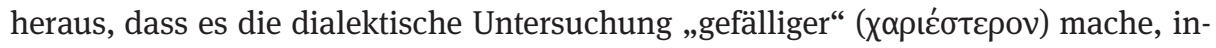
dem es die Schlussfolgerung des Gesagten einem anderen, nämlich dem Zitatautor, überlasse und die dialektische Präzision auflockere. Zugleich demonstriere dieses Zitat auch den richtigen Gebrauch von Dichterzitaten, die weder überladen noch zu lang sein sollten, sondern wie eine Parodie eine Varianz zum Prosaausdruck bieten sollte, ohne die Gattung des Textes zu überschreiten. ${ }^{107}$ Daher passt eine Passage aus der Stichomythie einer Tragödie gut zu einem philosophischen Dialog. Diese Leistung der Tragödie für die philosophische Argumentation liegt ja gerade in ihrer Eigenart begründet, die sinnliche Seite des Zuhörers anzusprechen.

Am beliebtesten ist bei Proklos das bereits bei Plotin verwendete Zitat aus den Troerinnen über den lautlosen, rechtmäßigen Gang des göttlichen Wirkens (Eur. Tro. $887 \mathrm{f}$.). Anders als Plotin gebraucht er allerdings die beiden Ausdrücke $\alpha \dot{\alpha} \varphi \omega$

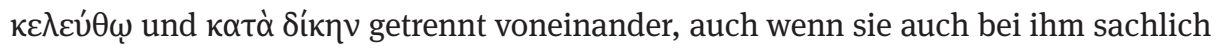
eine Einheit bilden. An den vielen Stellen, an denen es Proklos verwendet, werden damit diverse Aspekte des göttlichen Wirkens gekennzeichnet: ${ }^{108}$ Im Kontext einer

106 Wiederaufgenommen bei Olympiodor: Procl. In Alc. 292,1-7 bei Olymp. In Alc. 104,2-6; Procl. In Alc. 292,7-13 bei Olymp. In Alc. 101,19-102,3; vgl. Westerink 1954, 135.

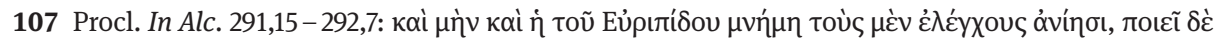

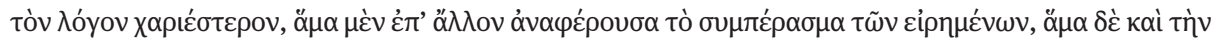

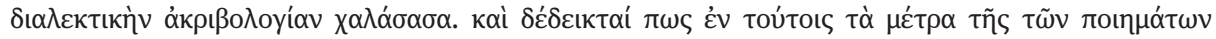

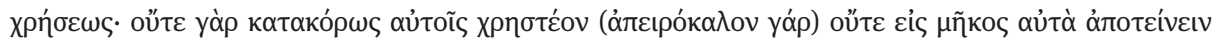

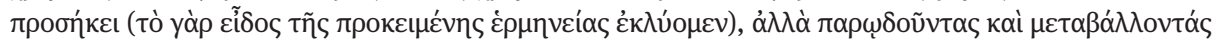

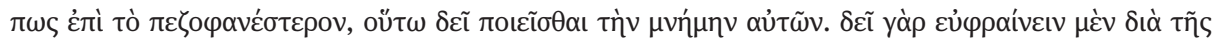

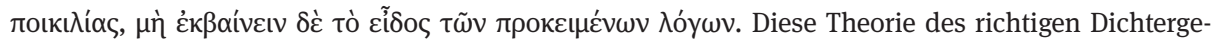

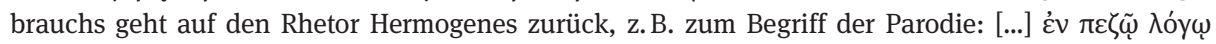

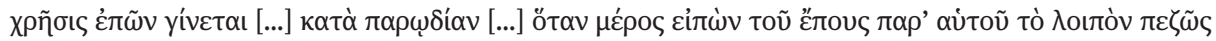

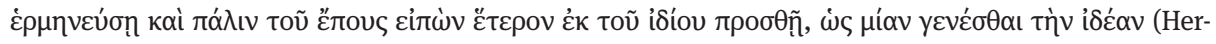
mog. Meth. 30, 447,5f. 17-20 Rabe; vgl. auch Olymp. In Gorg. 142,10 -12). Zum Einfluss des Hermogenes auf die Neuplatoniker vgl. Kustas 1973, 5-26.

108 Nicht auf die Theologie, sondern die Dialektik, die Logik und Methode der Gesprächsführung, bezogen ist die Verwendung des Zitats im Parmenides-Kommentar an der Stelle, wo Parmenides Sokrates durch Einführung eines geschickten Beispiels ,auf lautloser Bahn“ dazu bringt, ein bestimmtes Glied einer Disjunktion als Schlussfolgerung anzunehmen (Procl. In Parm. 4, 865,9-11). Dieser Zitatgebrauch steht in Übereinstimmung mit Proklos' eigener Maxime, dass ein Dichterzitat den dialektischen Untersuchungsgang auflockern solle (vgl. Procl. In Alc. 291,15-292,1). Zugleich macht es aber 
Erörterung der Theologie der Nomoi, wonach die Götter existieren, Pronoia für alles

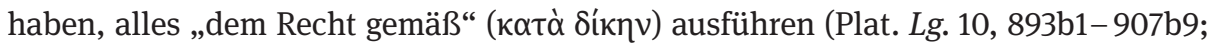
Procl. Theol. Plat. 1,13, 59,14-20 Saffrey-Westerink), stellt Proklos fest, dass die Götter

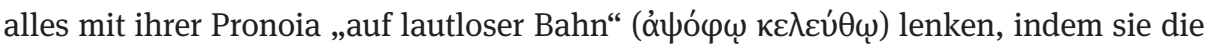
Ideen von allem in sich verwahren, aus sich das Sein von allem hervorgehen lassen und alles vollenden und mit Gütern anfüllen (Theol. Plat. 1,15, 75,3-8 Saffrey-Westerink). Das Zitat charakterisiert also die göttliche Pronoia als Eigenschaft der Demiurgie und Erhaltung des physischen Kosmos und seiner Einzeldinge durch die Götter. Dann

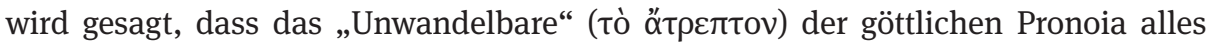

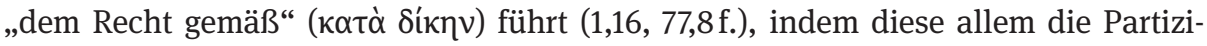
pation am Guten und somit die Ordnung des Ganzen ermöglicht (1,18, 87,17-21). ${ }^{109} \mathrm{Mit}$ dem Zitat wird also die Abkünftigkeit der Götter und des Kosmos vom höchsten Prinzip, dem transzendenten Guten, und damit die Gerechtigkeit innerhalb des noetischen, göttlichen und des physischen Kosmos begründet. Schließlich wird, ausge-

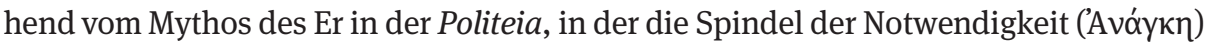
die Planetensphären bewegt und die Töchter der Notwendigkeit, die drei Parzen, die drei Modi der Zeit - Vergangenheit, Gegenwart und Zukunft - bestimmen (Plat. Rep. 10, 617a4-d1), die Ordnung des physischen Kosmos auf die Monade der Ananke und die Triade der Parzen zurückgeführt, wobei jene transzendent zu den enkosmischen Göttern steht, dem Himmel durch ihre Beständigkeit die Kreisbewegung eingibt und ihn „auf lautloser Bahn“ leitet (Theol. Plat. 6,23, 101,13-22 Saffrey-Westerink). ${ }^{110}$

Alle diese Aussagen differenzieren die bei Plotin einfach konstatierte Ordnung, Notwendigkeit und Gerechtigkeit des Kosmos in verschiedene Aspekte des göttlichen Wirkens, insbesondere der göttlichen Pronoia, in der Welt eigens aus, wobei diese durch das motivisch wiederkehrende Euripides-Zitat wie durch eine Klammer als zusammengehörig markiert und gleichsam wieder zur Einheit des Gedankens verbunden werden. Indem das Zitat für das Wirken von hierarchisch unterschiedlichen Götterstufen auf die Welt gebraucht wird, signalisiert es die Wohlordnung des gesamten Kosmos und Göttersystems.

auch deutlich, dass Parmenides hier als weiser Lehrer gegenüber einem Schüler in ähnlicher Weise wirkt wie die Götter auf den Kosmos (zur gottgleichen Stellung des Parmenides im Parmenides-Kommentar siehe Radke 2006, 306-346).

109 Mit der ersten Phrase des Zitats wird später (Procl. Theol. Plat. 4,14, 45,2-4 Saffrey-Westerink) ausgedrückt, dass die Götter durch die Gerechtigkeit das nach ihnen Kommende „auf lautloser Bahn“ leiten und das ihnen Zukommende zumessen. Das zeigt, dass die beiden Bestandteile des Zitats

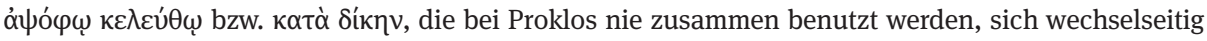
erläutern.

110 Ähnlich auch Procl. In Rep. 2, 227,3-5 zu Plat. Rep. 10, 617a5-b5. 


\subsection{Olympiodor}

Olympiodor, von dem Kommentare zum Großen Alkibiades, zu Gorgias und Phaidon, den ersten drei Dialogen im Platon-Curriculum der Neuplatonikerschule, ${ }^{111}$ sowie zu Aristoteles' Kategorien und der Meteorologie erhalten sind, spricht von Euripides schlechterdings als ò tpayıкós (In Alc. 30,12; In Gorg. 265,4 Westerink) oder als ì

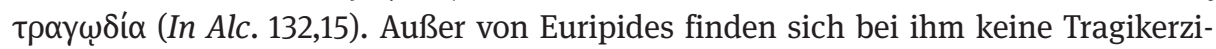
tate. ${ }^{112}$ Wie eine Zusammenfassung der entsprechenden Passagen in Proklos' Alkibiades-Kommentar lesen sich seine methodologischen Bemerkungen zum richtigen Gebrauch von Dichterzitaten anlässlich der Verwendung eines Euripides-Zitats (Hipp. 352) im Großen Alkibiades (113b8-c7): ${ }^{113}$ Man solle Dichterworte nicht ganz anführen, sondern nur einiges davon, um nicht Überdruss zu erregen und die Rede zu schwächen (In Alc. 104,3-6), und solle sie dem Prosakontext anpassen (In Gorg. 142,10 - 12 Westerink). Die deus ex machina-Technik der Tragödie - Olympiodor erinnert an den Apollon im Prolog der Alkestis - zieht er als Vergleich für die Einführung Gottes als fiktiven Gesprächspartner heran (In Alc. 44,4-7). Euripides-Sentenzen werden bei Olympiodor außerdem benutzt als Quelle für Sachinformationen, ${ }^{114}$ als literarische Illustration eines philosophischen Sprachgebrauchs ${ }^{115}$ oder als autoritative Bestätigung eigener Aussagen oder Interpretationen. ${ }^{116}$

Besonderer Beliebtheit erfreut sich bei ihm der Orestes: Den ersten Vers aus dem Prolog benutzt Olympiodor zur Illustration des tragischen Tons, den Sokrates an-

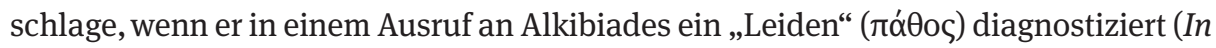
Alc. 132,15f. zu Plat. Alc. 1, 118b4). Das ,Leiden“ bestehe in einer „doppelten Unwissenheit“ ( $\left.\delta ı \pi \lambda \tilde{n} \alpha \dot{\alpha} \mu \theta^{\prime} \dot{\alpha}\right)$ - hinsichtlich seiner Seele sowie hinsichtlich seines Körpers und der äußeren Güter (In Alc. 170,5-7) -, die Sokrates „durch Widerlegung“ ( $\delta$ '”

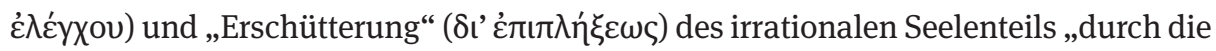

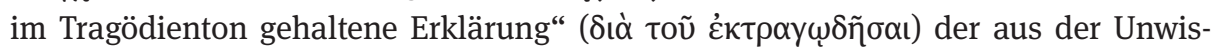

111 Vgl. Olymp. In Gorg. 6,1-6 Westerink; Festugière 1969, 287; O’Meara 2003, 63.

112 Mit Sophokles' Ajax, der namentlich genannt, aber nicht zitiert wird, und dessen Tötung der Schafe in der Meinung, sie seien Griechen (Aiax 18-65), wird Alkibiades verglichen, der das Gerechte zu wissen glaubt, es tatsächlich aber nicht weiß (In Alc. 103,21-23).

113 Die Stelle selbst wird, ähnlich wie bei Proklos, als Beitrag zur Erläuterung der sokratischen Gesprächstechnik verstanden, wonach der Antwortende selbst die Widerlegung vollziehen müsse, da Lernen „Wiedererinnerung“ ('́vón

114 Eur. Andr. 595-600 in In Alc. 166,20 f. (für Koedukation in Sparta, nicht wörtlich zitiert, aber mit Nennung des Euripides); Phoen. 1009-1014 in In Alc. 4,1 (für das Opfer des Menoikeus im Krieg zwischen Polyneikes und Eteokles; die Passage ist ebenfalls erwähnt in Ps.-Elias, In Isag. 15,25 Westerink). Auf frr. 182a, 484 und 839 dürfte sich In Meteor. 129,24-26 (über Erde und Himmel als physikalische Urprinzipien) beziehen.

115 Die Furien, „Mädchen, mit Blut bedeckt, wie der Tragiker sagt“ (Or. 256), zur Illlustration der Jenseitsstrafen (In Gorg. 265,1-5 zu Gorg. 524d7-527e7).

116 Eur. Andr. 696 in In Alc. 73,19-24; Hipp. 384 in In Alc. 139,18-20; Med. 245 in In Alc. 188,10 -12; Or. 395f. in In Alc. 23,9-13; Med. 410 in In Gorg. 25,8-10 Westerink; Eur. fr. 704 Kannicht in In Gorg. 235,1-7 Westerink. 
senheit folgenden Übel, z. B. Scham und Tadel, reinige (In Alc. 132,1-9 zu Plat. Alc.1, 117e9-118a9). ${ }^{117}$ Alkibiades, der glaubt, etwas zu wissen, es aber in Wirklichkeit nicht weiß, verhalte sich wie ein Arzt, der sich selbst aber für einen Philosophen hält, an den Alexander (von Aphrodisias oder von Tralles?) geschrieben habe: „Armer Kerl, bleib ruhig bei deinen Verbänden, du weißt nicht, was du sicher zu wissen glaubst“ ( $\mu \varepsilon ́ v \varepsilon, \tilde{\omega}$

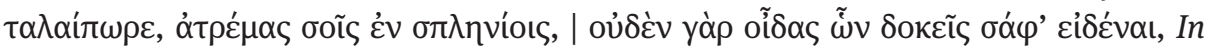
Alc. 170,8 -12). Dieses Zitat ist eine Variante zu Or. 258f., wo Elektra Orest, der sich von den Furien verfolgt sieht, mahnt, er möge ruhig „auf seinem Bett“ ('ُv $\delta \varepsilon \mu v i ́ o ı)$ bleiben, weil er nicht „sieht“ (òمõıৎ), was er sicher zu wissen glaubt: Die Diagnose der gestörten Sinneswahrnehmung ist also übertragen und angepasst auf den spezifisch sokratisch-platonischen Kontext von tatsächlichem und scheinbarem Wissen. ${ }^{118}$

Fast genauso häufig wie den Orest benutzt Olympiodor Zitate aus der Antiope, angeregt allerdings durch entsprechende Zitate in Platons Gorgias: Dort hatte Kallikles Sokrates davon zu überzeugen versucht, die Philosophie aufzugeben und sich der Politik zuzuwenden (484c4f., 485e3-486d1), und dies mit Zitaten aus der Antiope des Euripides illustriert. Wörtlich zitiert er das Fragment, wonach jeder sich am meisten darin müht, worin er am besten sei (Eur. Antiope fr. 184 Kannicht), und schließt daraus, dass die Philosophen sich in der Politik lächerlich machen würden und umgekehrt (Plat. Gorg. 484d7-485a3). Die Antiope war für Kallikles als Vergleichsfolie deshalb sehr interessant, weil darin Zethos, der ein soldatisches Leben führte, seinen Bruder, den Musiker Amphion, dazu ermahnt, das Leben eines Soldaten und Herrschers zu führen. Olympiodor stellt zu Recht heraus, dass Kallikles mit der Evokation dieser Parallele Sokrates dazu auffordere, statt Philosophie Politik zu treiben (Olymp. In Gorg. 141,32-142,4; 176,16-23 Westerink). ${ }^{119}$

Gegen Kallikles’ Plädoyer für einen ungezügelten Hedonismus und Immoralismus (Gorg. 491e5-492c8) hat Sokrates dann nach Olympiodor drei Argumente angeführt, nämlich ein Argument „aus der Meinung der Vielen“ („die Glücklichen bedürfen nichts“), eines „aus den Dichtern“ und eines „aus den Pythagoreern“ (einen pythagoreischen Vergleich der menschlichen Seele mit zwei Fässern, von denen das eine löchrig, d. h. zügellos, das andere dicht, d. h. maßvoll ist) (Gorg. 492e3-493d3; Olymp. In Gorg. 152,22-154,9 Westerink). Für das Dichterargument hat Sokrates das bekannte Euripides-Fragment angeführt: „Wer weiß, ob das Leben Sterben und das Sterben

117 Vgl. In Alc. 124,4-125,22. In diesem Kontext wird zur Erläuterung des Ausdrucks „tadelnswert“ Or. 4 („,der glückselige, ich tadle nicht das Schicksal“) benutzt (In Alc. 125,18-22).

118 Dass Olympiodor den originalen euripideischen Wortlaut kennt, zeigt sich In Gorg. 112,12-15

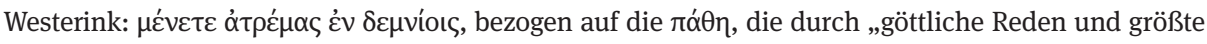
Gesänge“ zur Ruhe gebracht werden können.

119 Kallikles benutzt die Antiope in seiner Mahnrede an Sokrates im Gorgias noch an zwei weiteren Stellen mit wörtlichen, dem Kontext angepassten Zitaten, zu denen Olympiodor den korrekten Wortlaut des Euripides angibt, allerdings im ersten Fall nicht ganz korrekt und ohne eine Erklärung dieser Varianten vorzuschlagen (Olymp. In Gorg. 143,24-144,2 zu Plat. Gorg. 485e7-486a2, wo Eur. Antiope fr. 185,3f. Kannicht zitiert wird; Olymp. In Gorg. 144,8-10 zu Plat. Gorg. 486c4f., wo Eur. Antiope fr. 188,2f. Kannicht zitiert wird). 
Leben ist?“ (Eur. fr. 638 Kannicht). ${ }^{120}$ Sokrates hatte bereits gegen Kallikles den von ihm bevorzugten Dichter gewandt, und Olympiodor erläutert das Dichterwort, das in Sokrates' Argumentation nicht weiter kommentiert wird, im Ausgang von der platonischen Leib-Seele-Lehre, wonach die Seele dem Leib Leben eingebe, aber an dessen Sterblichkeit partizipiere und erst, wenn sie von diesem wieder getrennt sei, wieder wirklich lebe; sie sei hier eigentlich tot und müsse, weil der Körper daher die Ursache des Bösen sei, diesen zügeln (In Gorg. 153,12-19 Westerink).

\subsection{Medea-Rezeption bei Hierokles und Simplikios}

Das pythagoreische Carmen aureum und Epiktets Encheiridion gehörten zum ethischen Propädeutikum vor dem Aristoteles- und Platon-Studium. ${ }^{121}$ Daher ist es nicht überraschend, dass auch in Simplikios' Kommentierung von Epiktets Encheiridion ähnlich wie in diesem Werk selbst auch die euripideische Medea eine zentrale Stellung einnahm. Doch auch Hierokles von Alexandria nahm in seinem Kommentar zum pythagoreischen Carmen aureum zweimal Bezug auf die Medea: Als Beispiel zur Illustration des Verses, dass „es zu einem schlechten Menschen gehört, unvernünftig

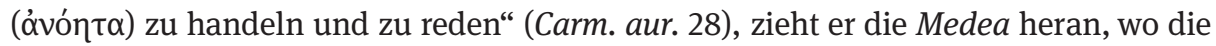
Titelfigur sich über die ihr widerfahrenen Ereignisse beklage, nachdem sie „schlechtberaten“ ( $\alpha \beta$ ov $\lambda \dot{\tau} \tau \varsigma)$ aus Liebe zu einem Fremden ihre Verwandten verraten und dieser sie dann verlassen habe, und sie sich dann - wörtlich wird Med. 144 zitiert - den Tod wünscht (In Carm. aur. 14,12, 66,17-25 Koehler). Hierokles kritisiert

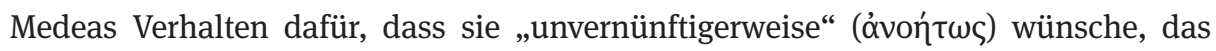
Geschehene sei nicht geschehen, und dann versuche, „Schlechtes mit Schlechtem zu

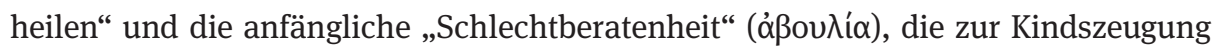
geführt habe, „durch ein schlechteres Ende“, die Tötung der Kinder, auszulöschen (67,1-5). Dies kulminiert in dem berühmten Zitat der Medea, sie erkenne, dass ihre

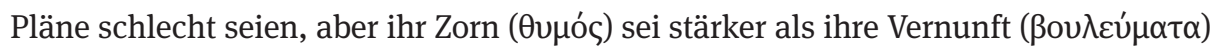
(Med. 1078f.; In Carm. aur. 8,4, 32,6f. Koehler). ${ }^{122}$ Hierokles analysiert diesen Widerspruch zwischen den verschiedenen Seelenteilen der Medea nicht weiter, sondern empfiehlt zum Schutz vor „solchen Dramen“ vielmehr die sokratische Maxime (Plat. Crit. 46b4-6), in allen Dingen dem Logos zu gehorchen, der nach rationaler Überle-

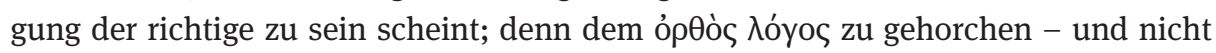

120 Dieses Zitat ist das einzige explizite Euripides-Zitat bei Iamblich (Protr. 17, 84,7-9 Pistelli) (Eur. fr. 1018 Kannicht ist fälschlich Hermotimos oder Anaxagoras zugewiesen, Iambl. Protr. 8, 48,15-17 Pistelli). In einem ähnlichen Kontext bei Hierokles wird Heraklit (DK 22 B 62) zitiert (Hierocl. In Carm. aur. 24,1, 98,7-9 Koehler).

121 Vgl. O'Meara 2003, $61 \mathrm{f}$.

122 Hierokles gibt zwei weitere Zitate: Das erste wiederholt die beiden Euripides-Verse und ersetzt

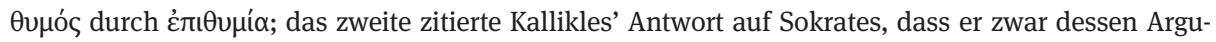
mentation gut, aber nicht überzeugend finde (Plat. Gorg. 513c4-6). 
dem nicht-rationalen Seelenteil - ist gleichbedeutend damit, Gott zu gehorchen (In Carm. aur. 14,15, 67,16-68,2 Koehler), was wiederum dem ethischen Ziel der Neupla-

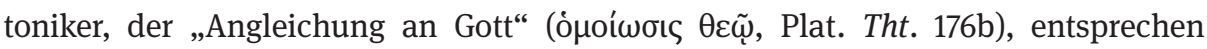
würde.

Während Hierokles die Medea des Euripides als Beispiel für schlechthin moralisch schlechtes Verhalten ansieht, weil sie ausschließlich der Unvernunft folge, steht

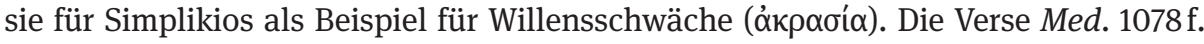
führt er in seinem Encheiridion-Kommentar dreimal an: Zuerst zitiert er sie zur Illustration des Fehlers ( $\alpha \mu \alpha ́ \rho \tau \eta \mu \alpha)$, wenn einer ,wenn auch schlaff, trotzdem sieht, was getan werden muss, die irrationalen Begierden sich aber aufgrund von Unbildung in

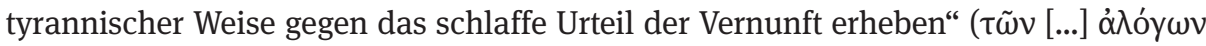

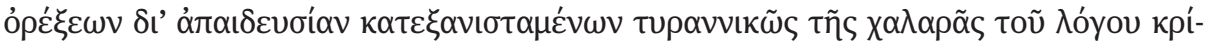

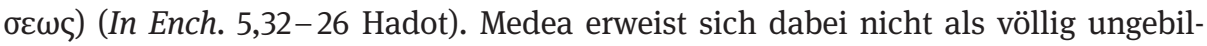
det, ${ }^{123}$ sondern nach der epiktetschen Unterscheidung als ein Anfänger in der Bildung, der sich selbst Vorwürfe macht, wenn es ihm schlecht geht (Epict. Ench. 5,7-9). Denn mit Med.1078f. gebe sie zu erkennen, wie Simplikios weiter erklärt, dass sie um Gut und Böse wisse, aber die Tyrannei der irrationalen Affekte in ihr ,aus Leichtfertigkeit, Trägheit der Vernunft und ununterbrochener Bewegung der Unvernunft“ ( $\delta ı \dot{\alpha} \rho \dot{\alpha} \theta \theta$ -

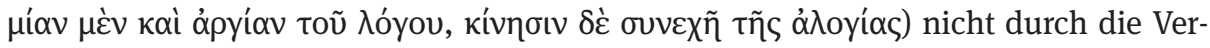
nunft gemäßigt sei (Simpl. In Ench. 11,19-29 Hadot). ${ }^{124}$ Sie finde sich also im Zustand

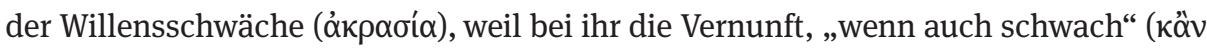
$\dot{\alpha} \mu \nu \delta \rho \tilde{\omega} \varsigma)$, das Richtige sehe, aber die irrationalen Affekte über diese die Oberhand hätten (12,50 - 60). ${ }^{125}$ Dem, der fehlerlos leben wolle, empfiehlt Simplikios - mit

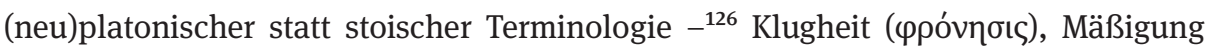

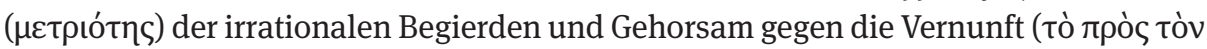

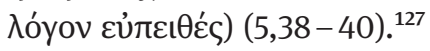

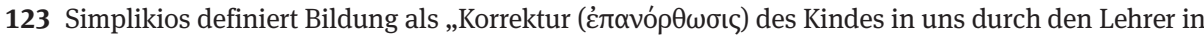
uns“, wobei das „Kind in uns“ die „Unvernunft“ ( $\alpha \lambda$ oý́ $\alpha)$, die nicht das Richtige sieht, sondern ausschließlich zum Lustvollen tendiert, und der „Lehrer in uns“ die „Vernunft“ ( irrationalen Affekte mäßigt (In Ench. 11,70 - 74).

124 Ähnlich in Simplikios' Kategorien-Kommentar, wo Med. 1078f. anlässlich einer Analyse von se-

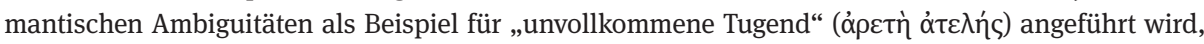
welche darin bestehe, dass nur eines der für vollkommene Tugend nötigen Elemente (nämlich $\lambda$ óyos,

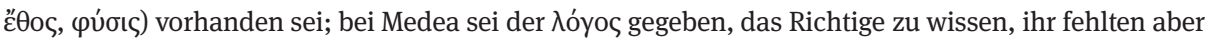

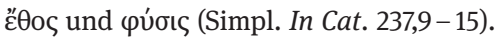

125 Ähnlich auch Elias In Cat. 180,11 f.; In Isag. 10,1-4; Ps.-Elias In Isag. 14,21 Westerink. Synesios benutzt Med. 1078f. innerhalb seines Fürstenspiegels zur Illustration der Seele, bei der die verschiedenen Teile nicht in Übereinstimmung miteinander stehen (Syn. Regn. 10, 22,11-21 Terzaghi). Bei Stobaios ist unter der Rubrik ópyń nicht Med.1078f., sondern 1079f. mit dem Fokus auf den $\theta v \mu o ́ \varsigma$ als

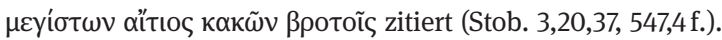

$126 \mathrm{Zu}$ Epiktets paränetischem Verfahren gegenüber der Medea vgl. oben S. $304 \mathrm{f}$.

127 Bei Simplikios ist von Euripides ansonsten nur fr. 1017 Kannicht zitiert (Simpl. In Phys. 328,1). 
Anders als Epiktet betrachten Hierokles wie Simplikios die Medea nicht als bemitleidenswerten Dialogpartner, dem - stellvertretend für alle Menschen - durch eine quasi-therapeutische Korrektur falscher Vorstellungen der rechte Vorstellungsgebrauch beigebracht werden soll. Sie ist vielmehr, ohne dialogisch-interaktive Einbettung, ein eindrückliches negatives Exempel bei Hierokles, um den Konnex von moralischer Schlechtigkeit und unvernünftigem Verhalten didaktisch zu illustrieren, während Simplikios an ihr das Phänomen der Willensschwäche als Widerspruch von rationalem und irrationalem Seelenteil verdeutlicht, wobei er ihr immerhin einen gewissen Grad von Selbsterkenntnis, intellektueller Einsicht und philosophischer Bildung zubilligt. Beide Kommentatoren tun dies vor dem Hintergrund des platonischsokratischen Intellektualismus.

\section{Schluss}

Bei den behandelten Autoren zeigt sich das ganze Spektrum der Euripides-Rezeption in den Philosophenschulen der Kaiserzeit und Spätantike: eine pädagogisch-paränetisch motivierte Nutzung bei Epiktet, die einerseits die Entscheidungsfreiheit des Menschen vorführt, andererseits als Ausgangspunkt für eine philosophische Korrektur dient; ein Residuum der eigenen Bildungsbiographie bei Marc Aurel, das im schreibenden Selbstdialog als Erinnerung an philosophische Grundsätze fungiert; eine argumentationstechnische Nutzung bei Sextus Empiricus, die zwar grundsätzlich kritisch gesehen, aber zusammen mit philosophischen Autoritäten als kumulative Evidenz für eine bestimmte philosophische Ansicht herangezogen wird; schließlich bei den Neuplatonikern die pädagogische Nutzung zur Vermittlung philosophischen, insbesondere ethischen Wissens im philosophischen Anfängerunterricht, die einerseits durch eine stringente systematische Interpretation der teilweise ambivalenten Äußerungen Platons zur Dichtung und ihre Einordnung in ein platonisches System des Wissens möglich geworden ist, andererseits die Praxis der Rhetorikschule in die Philosophenschule übernimmt und Tragödienzitate, teilweise angeregt durch Platons eigenes Verfahren in seinen Dialogen, zur didaktisch motivierten stilistischen Variation der philosophischen Diktion und rhetorischen Auflockerung der argumentativen Stringenz einsetzt.

Inhaltlich kommt es dabei zu bemerkenswerten intellektuellen Allianzen. Zugespitzt gesagt, ist der stoische Euripides des Epiktet ein sokratischer Euripides, der skeptische Euripides ein epikureischer Euripides und der neuplatonische Euripides ein platonischer Euripides. Teilweise unter Nutzung derselben Zitate kommen Stoa, Skeptizismus und Neuplatonismus zu divergierenden Ergebnissen: Für die Stoiker und die Neuplatoniker, die sich dabei teilweise auf die Stoa berufen, stehen die euripideischen Dramen als Mahnung zu oder Verwirklichung von Affektlosigkeit, der göttlichen Vernunftnatur des Menschen oder der Einsicht in die Vernünftigkeit der kosmischen Ordnung und der göttlichen Vorhersehung. Für die Skeptiker bürgt Euripides für die Unzulänglichkeit der menschlichen Erkenntnismöglichkeiten und die 
Indifferenz von Leben und Tod, während die Neuplatoniker letztere durch eine platonische Anthropologie deuten. Erstaunlicherweise verfolgen alle drei Schulen dasselbe Ziel: die Befreiung von äußeren Bedrängnissen, die die Stoiker und Neuplatoniker $\alpha \dot{\alpha} \alpha \dot{\theta \varepsilon \iota} \alpha$ und die Skeptiker in Anlehnung an die Epikureer $\alpha \dot{\tau} \alpha \rho \alpha \xi^{\prime} \alpha$ nennen. ${ }^{128}$ Insofern steht die Nutzung des Euripides durch Stoa, Skepsis und Neuplatonismus nicht nur für eine philosophische Rezeption, sondern für eine philosophische Nutzung spezifisch in der Kaiserzeit: sich angesichts der Unsicherheit der äußeren Welt auf sich selbst zu besinnen.

\section{Literatur:}

Bernard 1990: Wolfgang Bernard, Spätantike Dichtungstheorien. Untersuchungen zu Proklos, Herakleitos und Plutarch (Beiträge zur Altertumskunde 3), Stuttgart.

Biehl 1989: Werner Biehl, Euripides. Troades, erklärt, Heidelberg.

Blank 1998: David L. Blank, Sextus Empiricus, Against the Grammarians (Adversus Mathematicos I). Translated with an Introduction and Commentary, Oxford.

Bobzien 1997: Susanne Bobzien, „Stoic Conceptions of Freedom and their Relation to Ethics“, in: Richard Sorabji (Hg.), Aristotle and After, London, 71-89.

Bonhöffer 1894: Adolf Bonhöffer, Die Ethik des Stoikers Epiktet, Stuttgart (ND 1968).

Brandt 2015: Ulrike Brandt, Kommentar zu Epiktets Encheiridion, Heidelberg.

Brisson u. a. 1992: Luc Brisson u. a. (Hgg.), Porphyre, La vie de Plotin, vol. Il: Études d'introduction, texte grec et traduction française, commentaire, notes complémentaires, bibliographie, Paris.

Cilento 1960: Vincenzo Cilento, „Mito e poesia nelle Enneadi di Plotino“, in: Eric Robert Dodds (Hg.), Les Sources de Plotin. Dix exposés et discussions (Vandœuvres-Genève, 21 - 29 août 1957), Genf, 243-323.

Coulter 1976: James A. Coulter, The Literary Microcosm. Theories of Interpretation of the Later Neoplatonists (Columbia Studies in the Classical Tradition II), Leiden.

Dalfen 1967: Joachim Dalfen, Formgeschichtliche Untersuchungen zu den Selbstbetrachtungen Marc Aurels, Diss. München.

Dalfen 1971: Joachim Dalfen, „Das Gebet des Kleanthes an Zeus und das Schicksal“, Hermes 99, $174-183$.

Decleva Caizzi 1981: Fernanda Decleva Caizzi, Pirrone. Testimonianze, Neapel.

Dickey 2005: Eleanor Dickey, Ancient Greek Scholarship. A Guide to Finding, Reading and Understanding Scholia, Commentaries, Lexica, and Grammatical Treatises, from Their Beginnings to the Byzantine Period, Oxford.

Dillon 1997: John Dillon, „Medea among the Philosophers“, in: James J. Clauss u. Sarah I. Johnson (Hgg.), Medea: Essays on Medea in Myth, Literature, Philosophy, and Art, Princeton, 209-218.

Dobbin 1998: Robert F. Dobbin, Epictetus. Discourses Book I. Translated with an Introduction and Commentary, Oxford.

Dodds 1951: Eric Robert Dodds, The Greeks and the Irrational, Berkeley/Los Angeles.

Döring 1974: Klaus Döring, „Sokrates bei Epiktet“, in: ders. u. Wolfgang Kullmann (Hgg.), Studia Platonica, Amsterdam, 195-226.

128 Vgl. Epict. Diss. 1,4,27 = SVF 3,144; Epicur. Ep. Men. 131; Sext. PH 1,25-28. 
Egli 2003: Franziska Egli, Euripides im Kontext zeitgenössischer intellektueller Strömungen: Analyse der Funktion philosophischer Themen in den Tragödien und Fragmenten (Beiträge zur Altertumskunde 189), München/Leipzig.

Eliasson 2008: Erik Eliasson, The Notion of That Which Depends on Us in Plotinus and Its Background (Philosophia Antiqua 113), Leiden/Boston.

Farquharson 1944: Arthur S.L. Farquharson (Hg.), The Meditations of the Emperor Marcus Antoninus. Edited with Translation and Commentary, 2 vol., Oxford.

Festugière 1969: André-Jean Festugière, „L'ordre de lecture des dialogues de Platon aux Ve/Vie siècles“, Museum Helveticum 26, 281-296.

Gourinat 2001: Jean-Baptiste Gourinat, „Le Socrate d’Épictète“: Philosophe Antique 1, 137-165.

Kannicht 2004: Richard Kannicht, Tragicorum Graecorum Fragmenta (TrGF), vol. 5: Euripides, Göttingen.

Kuisma 1996: Oiva Kuisma, Proclus' Defence of Homer (Commentationes Humanarum Litterarum 109), Helsinki.

Kustas 1973, George L. Kustas, Studies in Byzantine Rhetoric (Analecta Vlatadou 17), Thessaloniki. Lamberton 1986: Robert Lamberton, Homer the Theologian. Neoplatonist Allegorical Reading and the Growth of the Epic Tradition, Berkeley/Los Angeles/London.

Lamberton 2012: Robert Lamberton, Proclus the Successor on Poetics and the Homeric Poems: Essays 5 and 6 of His Commentary on the Republic of Platon. Translated with an Introduction and Notes, Atlanta.

Lewy 2011: Hans Lewy, Chaldaean Oracles and Theurgy. Mysticism Magic and Platonism in the Later Roman Empire, Troisième édition par M. Tardieu avec un supplément „Les Oracles chaldaïques 1891-2011“, Paris.

Long 2002: Anthony A. Long, Epictetus. A Stoic and Socratic Guide to Life, Oxford.

Longo 2016: Angela Longo, „Senza far rumore“, la tragedia nella teodicea: una ripresa di Euripide (Troiane 887-888) in filosofi platonici di età imperial e tardoantica (Plutarco, Plotino, Proclo), Materiali e Discussioni per l'Analisi di Testi Classici 76, 213-228.

Marzillo 2010: Patrizia Marzillo, Der Kommentar des Proklos zu Hesiods „Werken und Tagen“. Edition, Übersetzung und Erläuterung der Fragmente (Classica Monacensia 33), Tübingen.

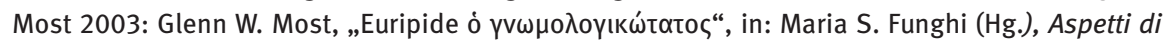
litterature gnomica, 2 Bde., Florenz, 141-166.

Nauck 1964: August Nauck, Tragicorum Graecorum Fragmenta, Editio secunda, Supplementum nova fragmenta Euripidea et adespota apud scriptores veteres reperta adiecit Bruno Snell, Hildesheim.

Nietzsche 1972: Friedrich Nietzsche, Die Geburt der Tragödie. Oder: Griechenthum und Pessimismus, in: Giorgio Colli u. Mazzino Montinari (Hgg.), Nietzsche, Werke. Kritische Gesamtausgabe, 3. Abt., Bd. 1, Berlin/New York, 5-152.

Nietzsche 1982: Friedrich Nietzsche, Philologische Schriften (1867-1873), in: Giorgio Colli u. Mazzino Montinari (Hgg.), Nietzsche, Werke. Kritische Gesamtausgabe, 2. Abt., Bd. 1, bearb. Fritz Bornmann u. Mario Carpitella, Berlin/New York.

Nussbaum 1993: Martha C. Nussbaum, „Poetry and the Passions: Two Stoic Views“, in: Jacques Brunschwig u. dies. (Hgg.), Passions and Perceptions. Studies in Hellenistic Philosophy of Mind. Proceedings of the Fifth Symposium Hellenisticum, Cambridge, 97-149.

O’Meara 2003: Dominic J. O’Meara, Platonopolis. Platonic Political Philosophy in Late Antiquity, Oxford.

Pappenheim 1881: Eugen Pappenheim, Erläuterungen zu des Sextus Empiricus Pyrrhoneïschen Grundzügen, Leipzig.

Patillon u. Segonds 1995: Michel Patillon u. Alain P. Segonds (Hgg.), Porphyre, De l'abstinence, Tome III, Livre IV. Texte établi, traduit et annoté, Paris. 
Perkams 2005: Matthias Perkams, „Stoische Schicksalslehre und christlicher Monotheismus. Kleanthes' Schicksalsverse im Spiegel ihrer Überlieferung“, in: Rosa Maria Piccione u. ders. (Hgg.), Selecta colligere, II: Beiträge zur Technik des Sammelns und Kompilierens griechischer Texte von der Antike bis zum Humanismus, Alessandria, 57-78.

Praechter 1899: Karl Praechter, „Zu Kleanthes Frgm. 91 Pears“, Archiv für Geschichte der Philosophie 12, $303 \mathrm{f}$.

Radke 2006: Gyburg Radke, Das Lächeln des Parmenides. Proklos' Interpretationen zur Platonischen Dialogform (Untersuchungen zur antiken Literatur und Geschichte 78), Berlin/New York.

Saffrey u. Westerink 1968-1997: Henri-Dominique Saffrey u. Leendert G. Westerink (Hgg.), Proclus, Théologie Platonicienne. Texte établi et traduit, vol. I-VI, Paris.

Sheppard 1980: Anne Sheppard, Studies on the $5^{\text {th }}$ and the $6^{\text {th }}$ Essays of Proclus' Commentary on the Republic (Hypomnemata 61), Göttingen.

Snell 1975: Bruno Snell, Die Entdeckung des Geistes. Studien zur Entstehung des europäischen Denkens bei den Griechen, Göttingen, 4. Aufl.

Tornau 1998: Christian Tornau, Plotin, Enneaden VI 4-5 [22-23]. Ein Kommentar, Stuttgart/Leipzig.

Van Ackeren 2011: Marcel van Ackeren, Die Philosophie Marc Aurels (Quellen und Studien zur Philosophie 103), 2 Bde., Berlin/Boston.

Warren 2015: James Warren, „Precursors of Pyrrhonism: Diog. Laert. 9.67-73“, in: Katja M. Vogt (Hg.), Pyrrhonian Skepticism in Diogenes Laertius. Introduction, Text, Translation, Commentary and Interpretative Essays by Katja M. Vogt, Richard Bett, Lorenzo Corti, Tiziano Dorandi, Christina M.M. Olfert, Elisabeth Scharffenberger, David Sedley, James Warren (SAPERE 25), Tübingen 2015, 105-121.

Wehner 2000: Barbara Wehner, Die Funktion der Dialogstruktur in Epiktets Diatriben (Philosophie der Antike 13), Stuttgart.

Westerink 1954: Leendert G. Westerink, Proclus Diadochus, Commentary on the First Alcibiades of Plato. Critical Text and Indices, Amsterdam.

Westerink u. Trouillard 1990: Leendert G. Westerink u. Jean Trouillard (Hgg.), Prolégomènes à la philosophie de Platon. Texte établi par L.G. W. et traduit par J. T., avec la collaboration de Alain P. Segonds, Paris. 